Historic, Archive Document

Do not assume content reflects current scientific knowledge, policies, or practices. 



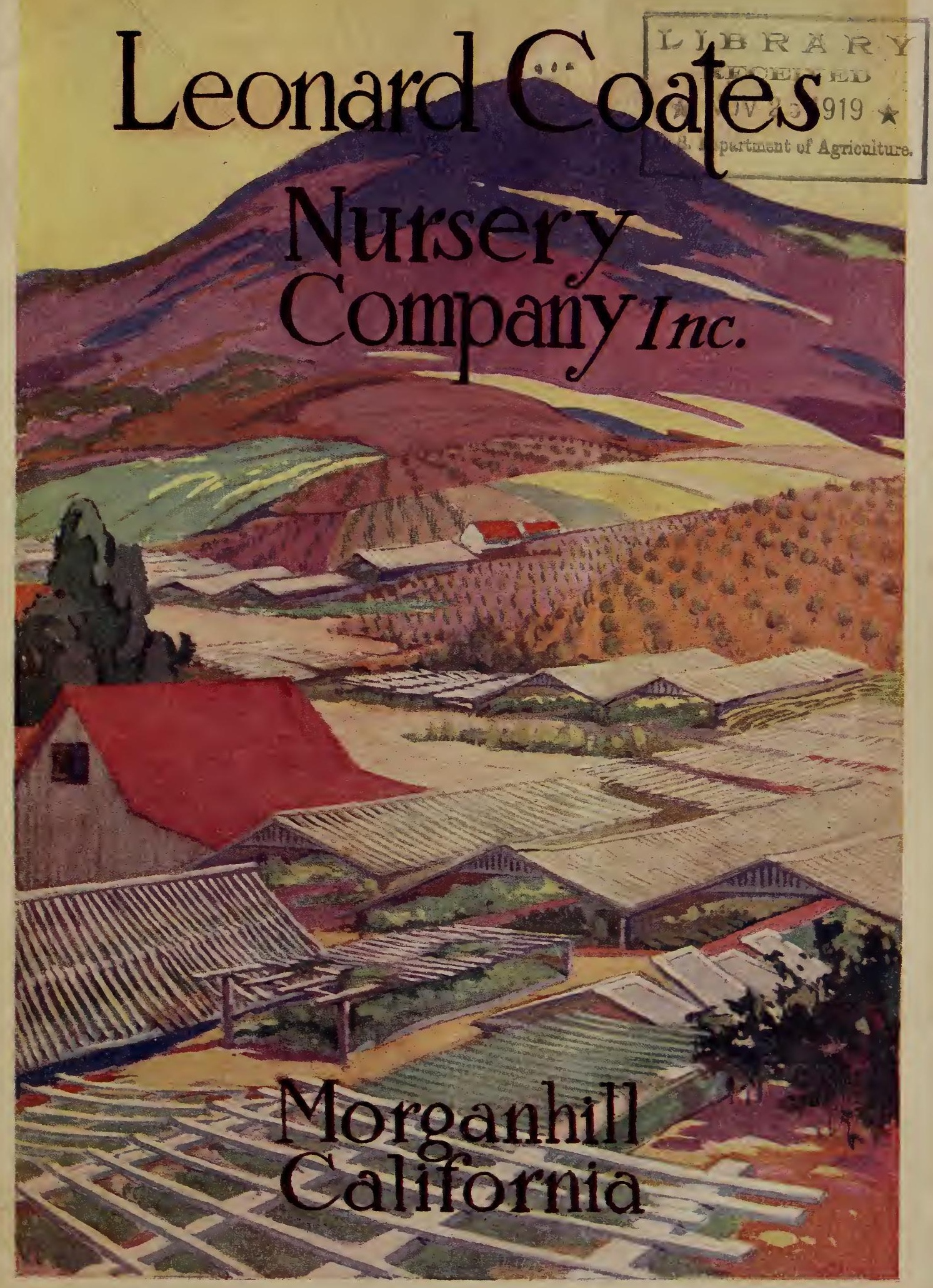


REAL ESTATE DEPARTMENT - Write for Particulars.

Ornamental Department arranged under common names

\section{GENERAL INDEX}

Page

Bamboo

Cannas

Climbing Plants

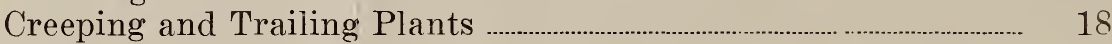

Deciduous Shade Trees ................................................................................. 8

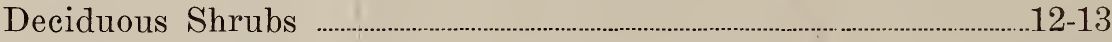

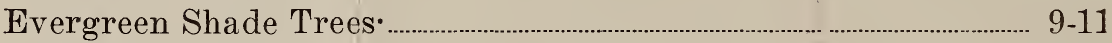

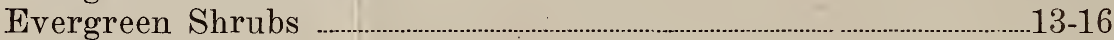

Fruit and Nut Trees …....................................................................................... 3- 4

Miscellaneous Fruits ………………………………………………………………. 5- 6

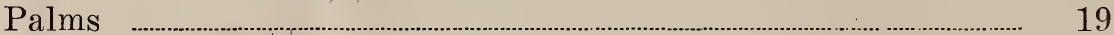

Perennials and Porch Plants .........................................................................17-18

Roses .................................................................................................... 19

\section{PLEASE NOTE.}

This catalog has no index other than the above. We have carefully rearranged the names of all our Trees, Shrubs, and Plants under Headings that will enable the reader to turn very quickly to any item. Common names are used.

\section{PLANT YOUR TREES PROPERLY}

This illustrates a Fruit Tree properly planted. Note the size and shape of hole and spread of roots at convex bottom. In a general way, observe the following. Dig holes at least $2 \frac{1}{2}$ $\mathrm{ft}$. wide by $2 \mathrm{ft}$. deep. Fill in with

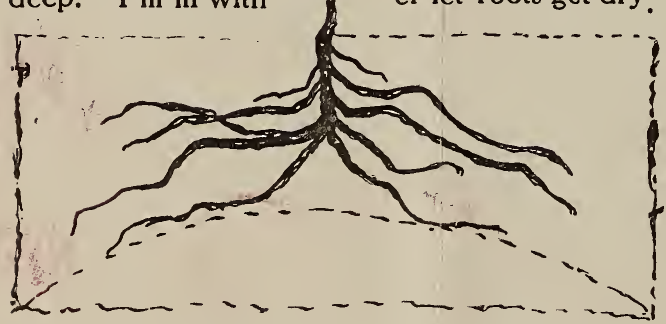

loose moist top-soil and press well below and around roots. Plant no deeper than tree stood in Nursery. Do not cut to less than 30 inches in height. Leave some laterals where possible. Caution! Never let roots get dry.

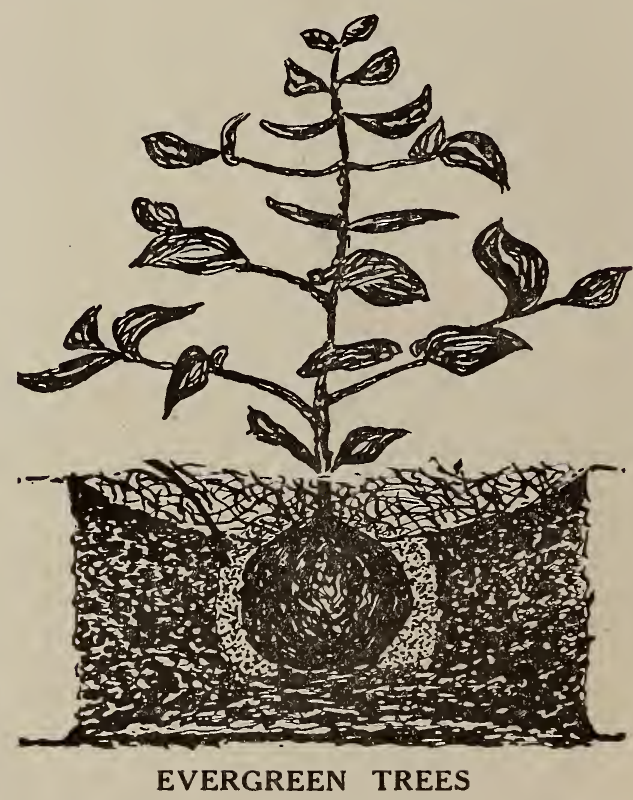

An Evergreen Tree properly planted. Dig hole as for a Fruit Tree; ball unbroken and fine earth pressed firmly below and around ball. Fill in of coarser earth and mulch of old manure in Basin. 
Remember. On receiving trees, open bale at once, cut open bunches, and spread roots in shallow trench; cover with sand or fine earth, and if weather is dry, soak the ground well.

If any trees should appear dry they may be soaked, root and top, in a trough all night.

While planting, keep roots moist by leaving them in a barrel half full of water or by "puddling", before taking to the field. This means to dip the roots into a hole previously prepared so that it is full of mud of the consistency of stiff paint; this will keep roots from drying quickly.

Shading. Shade the trunks with tree protectors or with burlap or paper; this is very important, as it prevents sunburn.

Shipping. Give plain directions as to route, and whether by freight or express. We recommend express for all except large shipments.

Parcels Post. When there is any saving to our customers we ship small packages by parcels post, if possible, postage and packing extra being charged.

Responsibility. We cannot be held liable for any damage that may result from delays in transportat on or through the inability of the horticultural officers to make prompt inspection.

Orders. We aim to fill orders in rotation as received, and will always endeavor to use the utmost promptness.

Subst:tutions. Always state, in a mixed order, if any substitutions may be made or not. If "no substitution" is written on order, such instructions will be carefully heeded.
Complaints. All claims for shortage, or any other possible mistakes, must be made within two days after receipt of stock or they cannot be allowed. Carefully examine trees or plants upon arrival. If anything is wrong, notify us at once; adjustments, if any are needed, are made at this time.

Guarantee of Genuineness. While every care is taken to sell stock true to name and description, mistakes may sometimes occur, in which case, on proper proof being presented, we shal be ready to refund the amount paid or will replace such trees or other stock.

It is mutually understood and agreed to between purchaser and ourselves that such guarantee of genuineness shall in no case make us liable for any sum greater than the original amount paid for any stock that may prove to be untrue. If this is not agreed to by purchaser, trees or plants must be returned at once.

Packing. This is done in the best manner, and charges made simply to cover cost of material used. No charge for cartage to railroad.

The charge per bale would amount to about $25 \mathrm{c}$ to $\$ 2.50$, according to size, and for ornamental stock, from $25 \mathrm{c}$ to $\$ 1.50$ per box. Small plants in flats, $15 \mathrm{c}$ each flat.

If any trees or plants are not satisfactory when received they must be returned, properly packed, within two days after they are received, as no claim on any account whatever will be allowed otherwise.

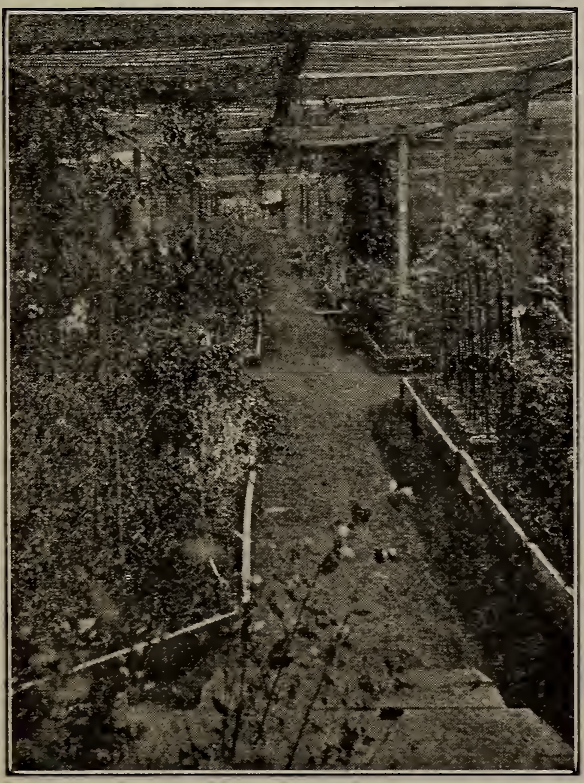

Vlew in one of our Lath Houses

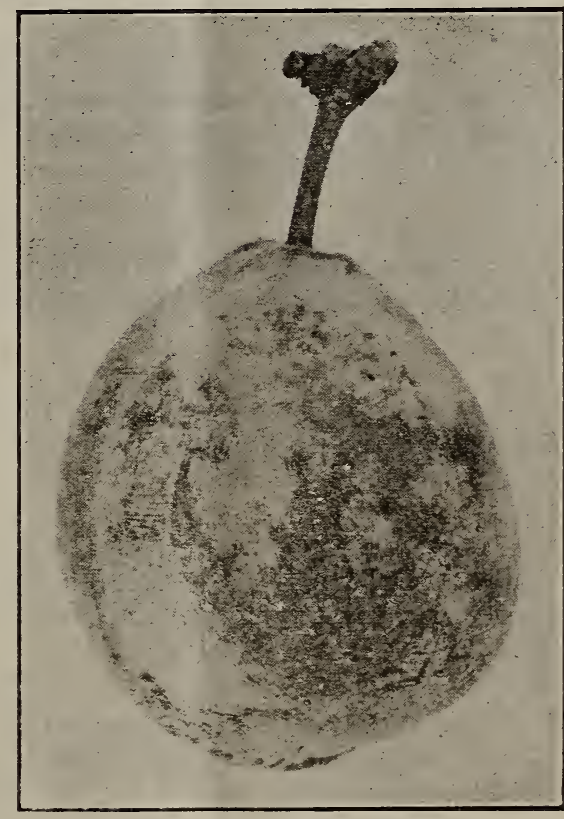

A true "sport" of the French Prune (Coates" French, 1418) or bud variant, one branch on an ordinary prune tree bearing this fruit and which has been reproduced several generations, show ing it to be a fixed type or variety 


\section{FRUIT TREE NOTES}

\section{APPLES}

The list comprises well-known and tried favorites, which may always be relied upon. We have a limited number of new and less known sorts, which we should be pleased to present with apple orders for trial, so far as the stock goes.

\section{ALMONDS}

We still propagate Lewelling in considerable quantity, simply because "it pays." This variety is the heaviest regular bearer of any. "Improved I. X. L." is a wonder, for size, appearance, quality, and bearing. Plant several varieties, the bees will do the rest

\section{APRICOTS}

Blenheim (Payne improved), Royal, Hemskirke (Flickinger) are the three most reliable commercial varieties. For the family orchard add one each of the others.

\section{CHERRIES}

Chapman, Tartarian, Royal Ann, Bing, Paul, are the best market sorts. For the family orchard a much larger assortment is desirable.

\section{PEACHES}

We grow a very superior assortment of varieties noted for their fine quality. Plant one of every kind, if you have room; it means ripe peaches daily from June to late October.

\section{PEARS}

No finer assortment in the U. S. For the small lot, many choice sorts may be had on quince root, which makes a smaller tree, precocious in bearing.

\section{PLUMS}

To appreciate the plum in a family orchard, a tree of as many as possible of our list should be used. The season extends for a full five months.

\section{PRUNES}

Our "Coates French Prunes" are standardized, which means much to the planter. This has been brought about by many years of patient and careful study and investigation. Full description is given on separate leaflet.

\section{PRUNING}

The University of California College of Agriculture is demonstrating a different method of pruning orchard trees. "Moderation" seems to be the key word, added to a selection at the start of three limbs to form the tree, all other growth eliminated as it starts, to give all the strength to these. Write to College of Agriculture, Berkeley, for full details.

Varieties of Fruit named as nearly as possible in order of ripening: 
THE demand for our largest prune-COATES FRENCH 1418-has been so great that all but a few were sold before this Catalog was ready to mail. We will sell the balance at the rate of two trees to a customer, to enable those who have none to try them out, if they wish to do so. We have commenced booking orders for 1920-21.

Leonard Coates Nursery Co. MORGANHILL, CALIF. 



\section{FRUIT TREES}

\section{APPLES}

Size-

Each.

.50

Per 10.

$\$ 4.50$

Per 100.

4-6 feet

.45

4.00

$\$ 45.00$

40.00

Named in order of ripening:

Yellow Transparent Alexander

Red Astrakan

Red June

Skinner Seedling

Alexander

Fameuse

King David

Gravenstein

Oldenburg

Banana
Stayman Winesap
Baldwin
Gano
Grimes Golden

Delicious

Northern Spy

Rome Beauty

Arkansas Black

Grimes Golden

Yel. Newton Pippin

CRAB APPLES

Yellow Siberian

Hyslop

\section{ALMONDS}

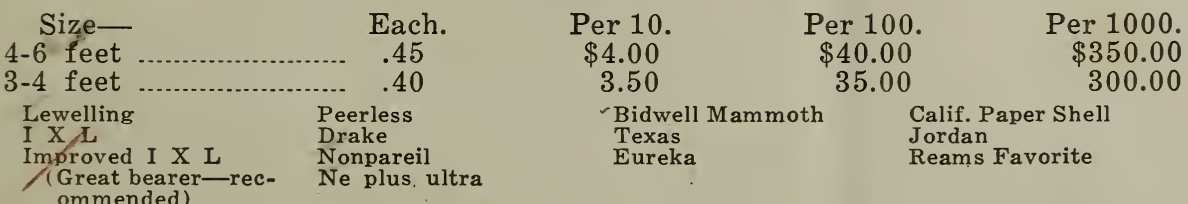

APRICOT

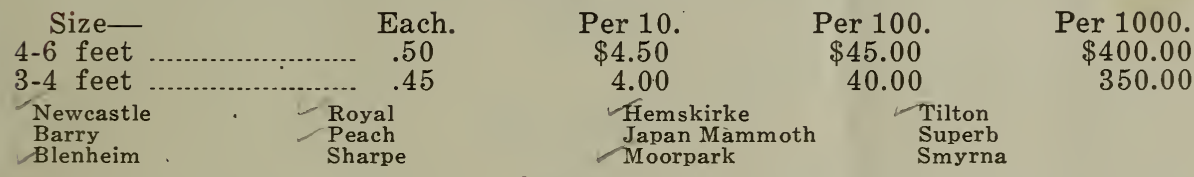

\section{CHERRY}

Named in order of ripening:

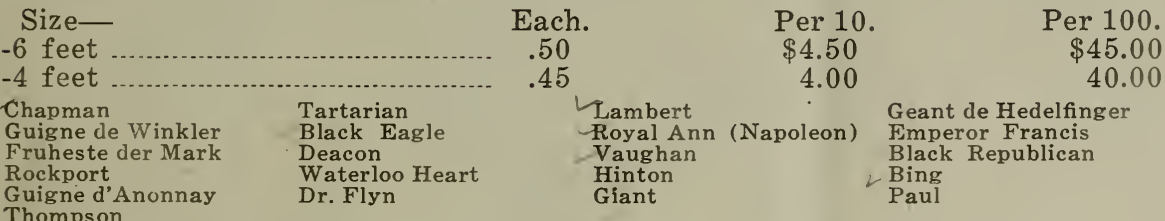

\section{FIG}

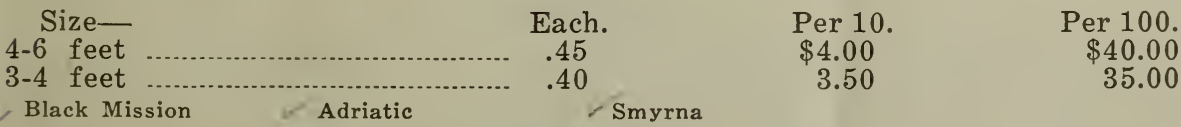

\section{PEACH}

Named in order of ripening.

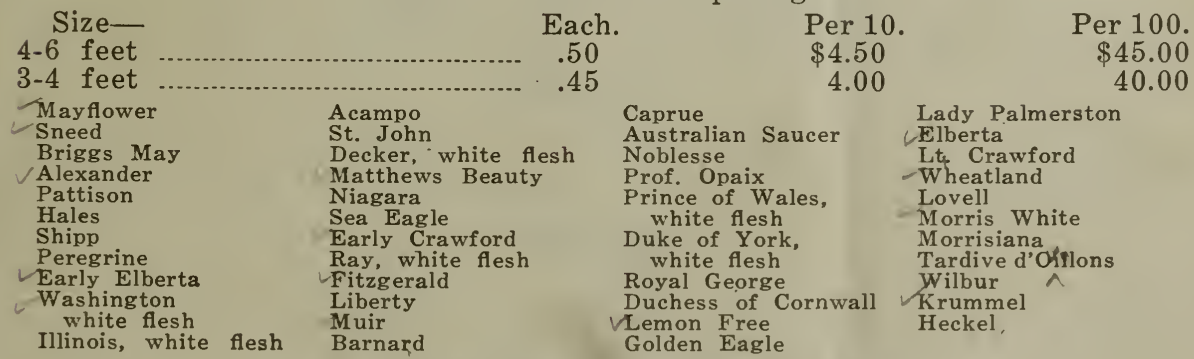

A Visit to Our Experimental Grounds is, "Worth While" 


\title{
CLING PEACH
}

\begin{tabular}{|c|c|}
\hline Tuscan & $\begin{array}{l}\text { Chillow } \\
\text { Japan Blood }\end{array}$ \\
\hline
\end{tabular}

\section{NECTARINES}

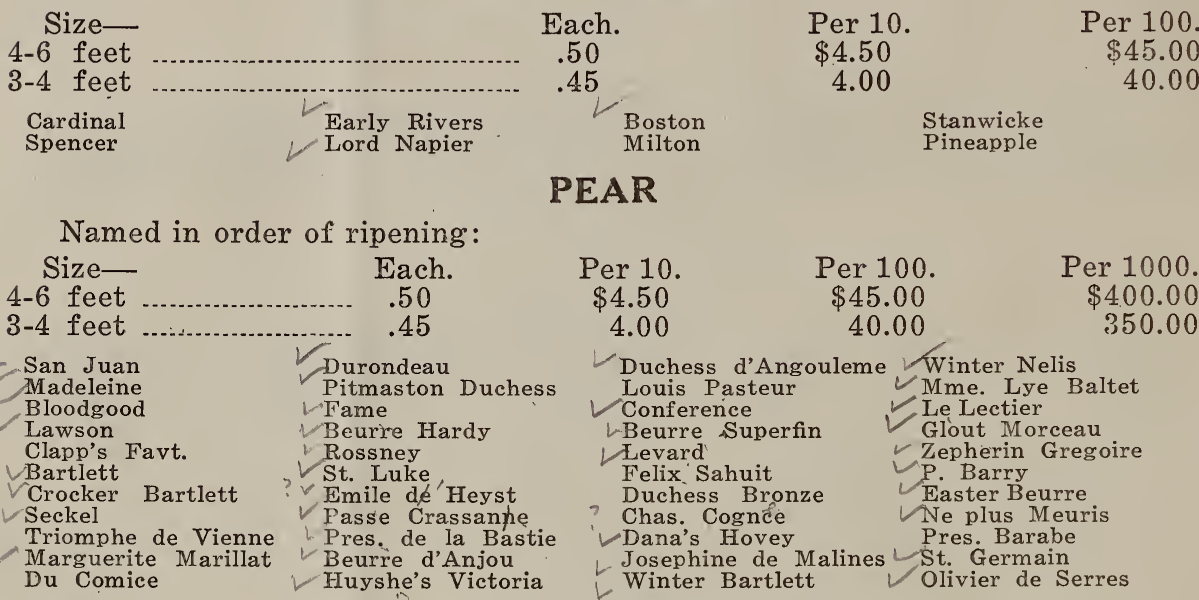

\section{PLUM}

Named in order of ripening:

Size-

4-6 feet

3-4 feet

Earliana (new)

Farly Satsuma (new) - Becky Smith

Clyman

Santa Rosa

Climax

Erna

Beauty

Gaviota

Methley

Wilma

Sultan

Satsuma onk (Cerulio Fraile, new)
Each

.50

.45

\section{- Hungarian \\ Decaisne}

Gigantic

Jefferson

Mammoth

Fernmonzi

Kelsey

Crimson Drop

Green Gage

\section{PLUMCOT}

\author{
Stanford \\ $\checkmark$ Sharpe
}

$50 \mathrm{c}$ each.

\section{PRUNE}

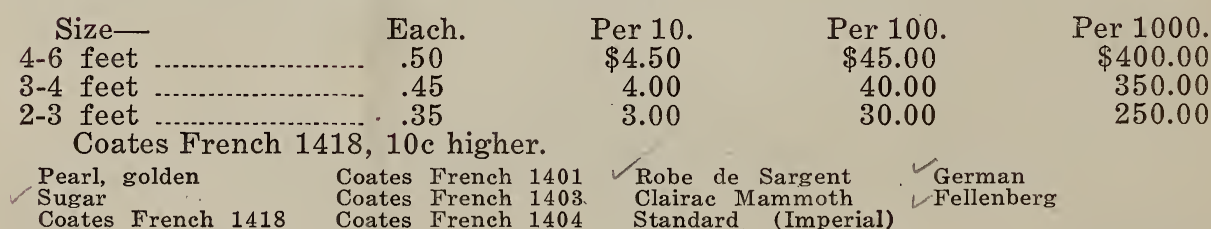

\section{OUINCE}

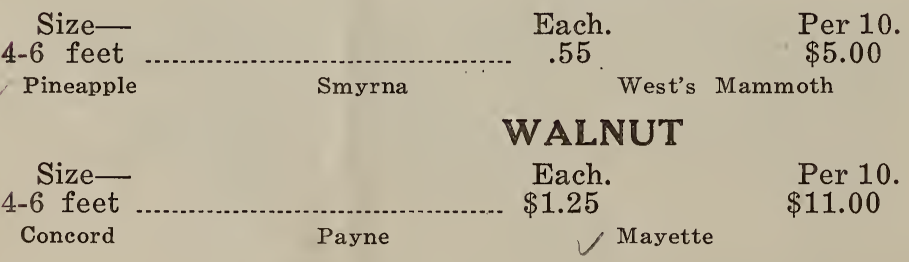

Per 10.

$\$ 4.50$

4.00

Per 100.

$\$ 45.00$

40.00

Conquest

Grand Duke

King of Damsons

Primate

Late Orange

President.

White Damson

Uncle Ben
45.00

0.00

0




\title{
JAPAN PERSIMMONS
}

Size-

2 yr. old, well rooted.

3-4 feet

Each.

$\$ 2.00$

Per 10.

Haychiya

$\$ 15.00$

\section{GRAPES}

\begin{tabular}{|c|c|c|c|c|}
\hline & $\begin{array}{l}\text { Each. } \\
.20\end{array}$ & $\begin{array}{l}\text { Per } 10 . \\
\$ 1.50\end{array}$ & $\begin{array}{l}\text { Per } 100 \\
\$ 10.00\end{array}$ & \\
\hline $\begin{array}{l}\text { Thompson Seedless } \\
\text { S-ltanina rosea } \\
\text { Rose of Peru }\end{array}$ & $\begin{array}{l}\text { Black I } \\
\text { Malaga } \\
\text { Dattier }\end{array}$ & routh $\frac{1}{1}$ & $\begin{array}{l}\text { Lady Finger } \\
\text { Flame Tokay } \\
\text { Muscat Hamburg }\end{array}$ & $\begin{array}{l}\text { Emperer } \\
\text { Muscat } \\
\text { Black Cornichon }\end{array}$ \\
\hline
\end{tabular}

\section{GRAPES. AMERICAN}

\begin{tabular}{ccc} 
Each. & Per 10. & Per 100. \\
.25 & $\$ 2.00$ & $\$ 15.00$ \\
Isabella & \multicolumn{1}{l}{ Niagara } &
\end{tabular}

\section{BLACKBERRY}

Mammoth, 20c ea., $\$ 1.50$ per 10 Himalaya, $20 \mathrm{c}$ ea., $\$ 1.50$ per 10 .

Humboldt (new), 25c ea.

Evergreen Thornless, $25 \mathrm{c}$ ea., $\$ 2.00$ per 10 .

\section{CURRANT}

\begin{tabular}{ccc} 
Each. & Per 10. & Per 100. \\
.25 & $\$ 2.00$ & $\$ 15.00$ \\
$\underbrace{}_{\substack{\text { Cherry, red } \\
\text { Black }}}$ & \multicolumn{2}{c}{$\begin{array}{c}\text { Perfection, red } \\
\text { White }\end{array}$} \\
\end{tabular}

GOOSEBERRY

$\begin{array}{ccc}\text { Each. } & \text { Per 10. } & \text { Per } 100 . \\ .25 & \$ 2.25 & \$ 20.00\end{array}$

Oregon Champion

\section{DEWBERRY}

$\begin{array}{cc}\text { Each. } & \text { Per } 10 . \\ .15 & \$ 1.25\end{array}$

LOGANBERRY
1 yr. transplants $\begin{aligned} & .20 \\ & .25\end{aligned}$
Each.
Per 10.
$\$ 1.50$
2.00
Per 100.
$\$ 10.00$
15.00

\section{RASPBERRY}

Belle de Fontenay, 25c ea.

Cuthbert, $15 \mathrm{c}$ ea., $\$ 1.25$ per 10 .

Hailsham, 25c ea.

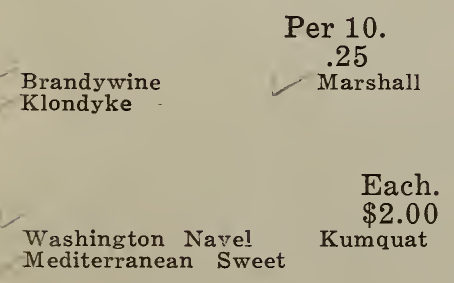

\section{STRAWBERRY}

\author{
Per 100. \\ $\$ 1.50$ \\ Banner
}

Per 1000 .

$\$ 10.00$

Magoon

\section{ORANGE}

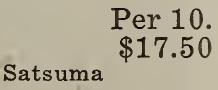

Valencia Late

\section{LEMON}

Each.

$\$ 2.00$

Eureka

Lisbon

Villa Franca

Per 10.

$\$ 17.50$

Let us Plan Your Ornamental Planting 


\section{LIME}

Bearrs' Seedless

$\$ 2.00$ each

\section{POMELO}

Marsh Seedless

$\$ 2.00$ each

\section{AVOCADO}

Seedlings

CAROB

Seedlings

.75 each

Italian or Spanish

\section{CHESTNUT}

\section{FEIJOA}

.50 to $\$ 1.00$ each.

\section{FILBERT}

.50 to $\$ 1.00$ each.

\section{HAZEL NUT}

.75 each.

GUAVA

$\checkmark$ Lemon

75 Strawberry

LOOUAT

.50 to $\$ 1.50$ each.

\section{MULBERRY}

Persian

OLIVE

In pots, 2-3 feet

\section{In pots, 3-4 feet}

Field grown, 3-4 feet

4-6 feet

Manzanillo

Mission

Sevillano

.50 to $\$ 1.00$ each

•

Budded varieties

PISTACHE

\section{POMEGRANATE}

.50 to $.7 \dot{5}$ each.

ARTICHOKE

Plants

\section{ASPARAGUS}

Per 10. .50

Per 100.

$\$ 4.00$

1.00 each 


\section{ORNAMENTAL DEPARTMENT}

\section{Please Note}

That we grow our own stock, and keep a large assortment of evergreen and flowering trees and shrubs in large tubs, boxes, or 5gal. cans, so that they may be transplanted at any time in the year. By this method, instead of "balling" a tree from the open ground, when the roots must be cut heavily, the planter gets a tree with roots undisturbed, growth proceeding as well as though the tree had not been removed.

We do not hesitate to say that there is no finer or healthier stock grown in the state, nor a more varied and useful assortment. Visitors express themselves as "amazed" when they see it.

We submit this condensed and classified list, arranged in a simple form, so that a selection may easily be made for any special purpose.

\section{THIS WILL BE THE SEASON FOR ORNAMENTAL PLANTING}

For several years, owing to war conditions, and the patriotic necessity for spending money only on essentials, owners of land and of town lots confined their plantings to fruit trees, berries, and vegetables.

The conditions have changed. Crops and prices are good, and most people have money to spend.

\section{DON'T FORGET}

that besides the satisfaction in having the home place beautified with trees and shrubs, it greatly enhances the value of any property to make such a planting.

\section{COME AND SEE}

our fine assortment of shrubs, plants and trees, and make your selection now. 


\section{DECIDUOUS SHADE TREES}

ACACIA NEMU, OR ALBIZZIA. Flowers pinkish. $4-6$ ft., 5 -gal. can, $\$ 1.00$ ea. $3-4 \mathrm{ft}$., 5-gal. can, $75 \mathrm{c}$ ea.

ALMOND, Flowering. Single white. Double white, pink, and red. 3-4 ft., 5-gal. can, $75 \mathrm{c}$ ea. 2-3 ft., 5-gal. can, $60 \mathrm{c}$ ea.

ALDER (California). 3-4 ft., 1-gal. can, 75c ea. ASH, American. $4-7 \mathrm{ft}$., bare root, $60 \mathrm{c}$ ea., $\$ 5.50$ per 10.

ASH, Arizona. Beautiful tree, used to arid conditions. $6-8 \mathrm{ft}$., bare root, $75 \mathrm{c}$ ea., $\$ 6.50$ per 10. 4-6 ft., bare root, $60 \mathrm{c}$ ea., $\$ 5.50$ per 10 .

ASH, Flowering (California) dwarf tree, or bush. 1-2 $1 / 2 \mathrm{ft}$., bare root, $60 \mathrm{c}$ ea.

APPLE, Bechtel's Flowering. 2-3 ft., 5-gal. can, $75 \mathrm{c}$ ea.

APPLE, Niedwitzkiana. Flowering, foliage purplish. 5-6 ft., 5-gal. can, $\$ 1.00$ ea.

BUCKEYE (California). 2-2 1/2 ft., cans, $40 \mathrm{c}$ ea., $\$ 3.50$ per 10 .

CATALPA, Western. Large leaves, fragrant lilac spotted flowers. 4-6 ft., bare root, $60 \mathrm{c}$ ea., $\$ 5.00$ per 10 .

CATALPA, Bungei. Round head; for formal planting. 4-6 ft., bare root, $\$ 1.50$ ea.

CHASTE TREE. Flowers blue; foliage graceful. 1-2 ft., pots, $50 \mathrm{c}$ ea. 2-3 ft., cans, $75 \mathrm{c}$ ea.

CHERRY, Flowering. Double white. 3-4 ft., pots, $60 \mathrm{c}$ ea. 5 -gal. cans, $75 \mathrm{c}$ ea.

CHERRY, Flowering. Double pink. 3-4 ft., pots, $60 \mathrm{c}$ ea. 3-4 ft., 5-gal. can, $75 \mathrm{c}$ ea.

CHERRY, Chinese. Flowering; foliage large. 3-4 ft., 5-gal. can, $75 \mathrm{c}$ ea.

CHESTNUT, Italian. 2-3 ft., bare root, $50 \mathrm{c}$ ea.

CHINA TREE (Koelreuteria). Flowers yellow: pods crimson. 3-4 ft., bare root, $60 \mathrm{c}$ ea. 4-6 ft., bare root, $75 \mathrm{c}$ ea. 4-6 ft., 5-gal. can, $\$ 1.25$ ea.

CHOKE CHERRY (California). Beautiful in bloom. 3-4 ft., bare root, $60 \mathrm{c}$ ea.

COFFEE BEAN. 1-2 ft., pot, $25 \mathrm{c}$ ea.

CRAPE MYRTLE. Pink or crimson flowers. $8-12$ in., pot, $35 \mathrm{c}$ ea. $1-2 \mathrm{ft}$., pot, $50 \mathrm{c}$ ea. $2-3$ ft., pot, $75 \mathrm{c}$ ea.

DESERT WILLOW (Chilopsis) California. Large tubular flowers. 1-1 1/2 ft., pot, $50 \mathrm{c}$ ea., $\$ 5.00$ per 10. 3-4 ft., bare root, $75 \mathrm{c}$ ea., $\$ 6.50$ per 10 .

ELM, Weeping. 6-8 ft., box, $\$ 4.00$ ea.

GOLDEN CHAIN (Laburnum). 6-7 ft., bare root, $75 \mathrm{c}$ ea.

INDIGO TREE, California (Amorpha). Locustlike leaves; flowers in long, compact racemes; purple and gold. 4-6 ft., 5-gal. can, $75 \mathrm{c}$ ea.

ELDER, Golden. 1-2 $1 / 2 \mathrm{ft}$., pots, $50 \mathrm{c}$ ea., $\$ 4.00$ per 10. Red-Berried. 6-in.-1 ft., pots, $50-75 \mathrm{c}$ ea. Common. $3 \mathrm{ft}$, bare root, $50 \mathrm{c}$ ea. Burbank; berries white. $1 \mathrm{ft}$., pots, $50 \mathrm{c}$ ea.

HACKBERRY (California). Spreading; berries orange red. 4-6 ft., bare root, $75 \mathrm{c}$ ea.

HAWTHORN (Crataegus coccinea). Red berries. $2-3 \mathrm{ft}$, bare root, $60 \mathrm{c}$ ea., $\$ 5.00$ per 10 . Red berries. $3-4 \mathrm{ft}$., pots, $75 \mathrm{c}$ ea., $\$ 6.00$ per 10 .

HAWTHORN. Crus-galli, red berries. 2-3 ft., bare root, $60 \mathrm{c}$ ea., $\$ 5.00$ per 10 . 3-4 ft., bare root, $75 \mathrm{c}$ ea., $\$ 6.00$ per 10 .

These are two handsome species, quite hardy, which should be largely planted.

HAWTHORN, Double-Scarlet flowers. 6-7 ft., 5 -gal. can, $\$ 1.25$ ea. $4-6 \mathrm{ft}$., bare root, $65 \mathrm{c}$ ea., $\$ 5.00$ per 10 .

HAWTHORN. Pink flowers. 5-6 ft., 5-gal. can,

$\$ 1.25$ ea.
HAWTHORN. White flowers. $8-10 \mathrm{ft}$., 5 -gal. can, $\$ 3.50$ ea. $3-4 \mathrm{ft}$., 5 -gal. can, $75 \mathrm{c}$ ea. $5-6$ ft., 5 -gal can, $\$ 1.25$ ea.
HAWTHORN. Single White flowers. $6-10$ in., bare root, $20 \mathrm{c}$ ea., $\$ 1.50$ per $10.2-3 \mathrm{ft}$., bare root, $30 \mathrm{c}$ ea., $\$ 2.50$ per 10 . $3-4 \mathrm{ft}$., bare root, $40 \mathrm{c}$ ea., $\$ 3.00$ per 10 . 4-6 ft., bare root, $50 \mathrm{c}$ ea., $\$ 4.00$ per 10

Very useful under shade of Oak trees, trees covered with red berries.

JAPAN PAGODA TREE. (Sophora). Beautiful tree, weeping habit; leaves dark green, flrs. white, in large pannicles. In 5-gal. cans, 5-6 ft., $\$ 1.50$ ea.

LARCH. Tamarack. Succeeds well in California. 5-6 ft., 5-gal. can, $\$ 1.50$ ea. $4-5$ ft., 5-gal. can, $\$ 1.25$ ea.

LINDEN, American. 4-6 ft., bare root, 50c ea. European. $3 \mathrm{ft}$., bare root, $50 \mathrm{c}$ ea.

LOCUST, Robinia. Late pink flowering. 4-5 ft., bare root, $\$ 1.00$ ea.

LOCUST, Hispida. Rose colored. 4-6 ft., bare root, $\$ 1.00$ ea.

LOCUST, Langleyi. Pink, compact; foliage reddish; rare. 3-4 ft., bare root, $\$ 1.50$ ea.

LOCUST, Viscosa. Light pink, compact. 3-5 ft., bare root, $\$ 1.00$ ea.

MAPLE, Broad-leaved. (California). 6-8 $\mathrm{ft}$. bare root, $75 \mathrm{c}$ ea., $\$ 6.50$ per 10 .

MAPLE, Box Elder (California). 6-8 ft., bare root, $75 \mathrm{c}$ ea.

MAPLE, English. 3-4 ft., bare root, $50 \mathrm{c}$ ea. $\$ 4.50$ per $10.4-6 \mathrm{ft}$., bare root, $75 \mathrm{c}$ ea., $\$ 6.50$ per 10. 6-7 ft., bare root, $\$ 1.00$ ea., $\$ 8.50$ per 10. Very adaptive; holds foliage late.

MAPLE, Dwarf (California). $1 \mathrm{ft}$., bare root,

$75 \mathrm{c}$ ea.
MAPLE, Japanese. 2-3 ft., pot, $75 \mathrm{c}$ ea.

MAPLE, Silver. Succeeds well in California. $5-6 \mathrm{ft}$., bare root, $60 \mathrm{c}$ ea., $\$ 5.00$ per 10 . $6-8$ ft., bare root, $75 \mathrm{c}$ ea., $\$ 7.00$ per 10 .

MAGNOLIA PURPUREA. Immense purple flowers. 6-8 ft., 5-gal. can, $\$ 4.00$ ea.

MONTEZUMA CYPRESS (Taxodium mucronatum). Rare; "Evergreen Bald Cypress", 4-5 ft., 5-gal. can, $\$ 2.50$ ea.

MOUNTAIN ASH. 4-5 ft., bare root, $40 \mathrm{c}$ ea.

MULBERRY, Persian; large fruit. 3-4 ft., bare root, $75 \mathrm{c}$ ea. 4-6 ft., bare root, $\$ 1.00$ ea.

OAK, Quercus lobata. Valley Oak. $2-2 \frac{1}{2}$ ft., pot, $60 \mathrm{c}$ ea.

PEACH, Flowering. Double, red. 3-4 ft., pot, $75 \mathrm{c}$ ea. 4-6 ft., 5-gal. can, $\$ 1.00$ ea. White 3-4 ft., pot, $75 \mathrm{c}$ ea. $4-6 \mathrm{ft}$., 5 -gal. can, $\$ 1.00$ ea.

PLANE, European. 4-6 ft., bare root, $60 \mathrm{c}$ ea.

POPLAR, Lombardy. 5-6 ft., 5-gal. can, 50c ea., $\$ 4.00$ per 10 . 6-8 ft., 5 -gal. can, $75 \mathrm{c}$ ea., $\$ 6.00$

per 10 . Norway. 4-6 ft., bare root, $40 \mathrm{c}$ ea.
POPLAR, Norwat,

POPLAR, Silver. 4-6 ft., bare root, $50 \mathrm{c}$ ea., $\$ 4.00$ per $10.6-7 \mathrm{ft}$., bare root, $65 \mathrm{c}$ ea., $\$ 6.00$ per 10.

PLUM, Flowering. (Prunus triloba). 4-5 ft., 5-gal. can, $\$ 1.00$ ea. ( $P$. emarginata-California) small tree, $1-2 \mathrm{ft}$., bare root, $60 \mathrm{c}$ ea. Purple-leaved. 4-6 ft., bare root, $50 \mathrm{c}$ ea. Vesuvius, large purple leaves. 4-6 ft., bare root, $50 \mathrm{c}$ ea.

RED-BUD (Cercis occidentalis-California). 3-4 $\mathrm{ft}$., bare root, $60 \mathrm{c}$ ea., $\$ 5.50$ per 10 . $4-6 \mathrm{ft}$., bare root, $75 \mathrm{c}$ ea., $\$ 7.00$ per 10 . (C. Canadensis). 4-6 ft., bare root, $75 \mathrm{c}$ ea., $\$ 7.00$ per 10 .

WALNUT, Black California. 3-4 ft., bare root, $40 \mathrm{c}$ ea., $\$ 3.00$ per 10 . 4-6 ft., bare root, $50 \mathrm{c}$ ea., $\$ 4.00$ per 10

WILLOW, Weeping. 4-6 ft., bare root, $50 \mathrm{c}$ ea.,

PAULONIA IMPERIALIS. Immense leaves, blue flowers. 6-8 ft., bare root, $\$ 1.00$ ea.

PECAN. Very shapely, handsome avenue tree. 2-3 ft., $40 \mathrm{c}$ ea. 


\section{EVERGREEN SHADE TREES}

ABERIA. Small African tree; bears edible fruit. 1-2 ft., pots, $50 \mathrm{c}$ ea.

ACACIA ARMATA. Small, holly-like leaves, yellow flowers. 1-2 ft., pots, $40 \mathrm{c}$ ea.

A. BAILEYANA. Bluish, feathery foliage, yel-

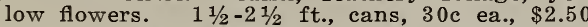
per 10 . $1 \frac{1}{2}-2 \frac{1}{2} \mathrm{ft}$., pots, $35 \mathrm{c}$ ea., $\$ 3.00$ per 10. $2-3 \mathrm{ft}$., pots, $40 \mathrm{c}$ ea., $\$ 3.50$ per 10 . $3-4$ ft., pots, $60 \mathrm{c}$ ea., $\$ 5.50$ per 10 . 4-6 ft., pots, $75 \mathrm{c}$ ea., $\$ 7.00$ per 10 .

A. CULTRIFORMIS. Usually trained as a shrub; covered with yellow flowers. $1-2 \mathrm{ft}$. pots, $30 \mathrm{c}$ ea., $\$ 2.50$ per 10 . $2-3 \mathrm{ft}$., pots, $40 \mathrm{c}$ ea., $\$ 3.50$ per 10 .

A. CYCLOPS. Low growing; new. 1-2 ft., pots, $40 \mathrm{c}$ ea.

A. DEALBATA. Large, spreading; flowers yellow. $6-8 \mathrm{ft}$., 1 gal. can, $\$ 1.00$ ea., $\$ 8.50$ per 10 .

A. DECURRENS. Large tree; flowers nearly white, late. $2-3 \mathrm{ft}$., pot, $25 \mathrm{c}$ ea., $\$ 2.50$ per 10 . $3-4 \mathrm{ft}$., pot, $40 \mathrm{c}$ ea., $\$ 3.50$ per 10 . $4-6 \mathrm{ft}$, pot, $60 \mathrm{c}$ ea., $\$ 5.00$ per 10.

A. FLORIBUNDA; long narrow leaves; flowers all summer. $21 / 2-3 \frac{1}{2} \mathrm{ft}$., pot, $35 \mathrm{c}$ ea., $\$ 3.00$ per $10.31 \frac{1}{2}-5 \mathrm{ft}$., pot, $40 \mathrm{c}$ ea., $\$ 3.50$ per 10 .

A. EXTENSA. Very narrow leaves. Small plants, pot, $35 \mathrm{c}$ ea., $\$ 2.50$ per 10

A. FARNESIANA, thorny. Flowers used for perfume; deep yellow. 4-6 ft., 1-gal. can, $60 \mathrm{c}$ ea., $\$ 5.00$ per 10 .

A. JUNIPERINA, narrow, spiny leaves. $2 \mathrm{ft}$., pot, $50 \mathrm{c}$ ea.

A. LÁTIFOLIA. 2-3 ft., pot, $35 \mathrm{c}$ ea., $\$ 3.00$ per 10. $3-4$ ft., pot, $40 \mathrm{c}$ ea., $\$ 3.50$ per 10 . 4-6 ft., pot, $60 \mathrm{c}$ ea., $\$ 5.00$ per 10 .

A. LINIFOLIA. Slender tree; new. $10-20$ in., pot, $50 \mathrm{c}$ ea.

A. MELANOXYLON. Black wattle. 3-4 ft., pot, $40 \mathrm{c}$ ea., $\$ 3.50$ per 10 . $4-6 \mathrm{ft}$., pot, $60 \mathrm{c}$ ea. $\$ 5.00$ per 10 .

A. MOLLISSIMA. Large tree. 2-3 ft., pot, $35 \mathrm{c}$ ea., $\$ 3.00$ per 10 . $4-6 \mathrm{ft}$., can, $50 \mathrm{c}$ ea., $\$ 4.00$ per 10.

A. NORMALIS; new. 2-3 ft., pot, $50 \mathrm{c}$ ea

A. PODALYRAEFOLIA. 1-2 ft., pot, 50c ea.

A. PRAVISSIMA. One of the very best. 2-3 $\mathrm{ft}$, pot, $40 \mathrm{c}$ ea., $\$ 3.00$ per $10.3-4 \mathrm{ft}$., pot, $60 \mathrm{c}$ ea., $\$ 5.00$ per 10 .

A. PRUINOSA. 2-3 ft., pot, $50 \mathrm{c}$ ea.

A. PYCNANTHA. 1-2 ft., pot, $35 \mathrm{c}$ ea.

A. SALIGNA. $1-21 / 2 \mathrm{ft}$, pot, $50 \mathrm{c}$ ea.

A. VERTICILLATA. $1-2 \mathrm{ft}$, pot, $35 \mathrm{c}$ ea., $\$ 3.00$ per 10 . 2-3 ft., pot, $50 \mathrm{c}$ ea., $\$ 4.00$ per 10 . 3-4 ft., 1 gal. can, $75 \mathrm{c}$ ea.

ARAUCARIA BIDWILLII. 1-11/2 ft., pot, $\$ 1.50$ ea. 2-3 ft., pot, $\$ 2.00$ ea.

ARBOR VITAE (Thuja). 1-2 ft., pot, $50 \mathrm{c}$ ea. 5-6 ft., pot, $\$ 1.00$ ea. $2-3$ ft., 5 -gal. can, $\$ 1.50$ ea. $3-4 \mathrm{ft}$., 5 -gal. can, $\$ 2.50$ ea. $5 \mathrm{ft}$., 5 -gal. can, $\$ 3.50$ ea.

BAY TREE. (Laurus nobilis). $3-4$ ft., 5-gal. can, $\$ 1.75$ ea. 5.ft., 5-gal. can, $\$ 3.50$ ea. $2-3$ ft., pot, $\$ 1.00$ ea., $\$ 8.50$ per $10,3-4 \mathrm{ft}$., pot, $\$ 1.25$ ea.

BIG TREE (Sequoia gigantea). $2 \mathrm{ft}$., pot, $\$ 1.50$ ea., $\$ 12.50$ per 10 . 1-2 ft., can, $75 \mathrm{c}$ ea. Large plants. 2 ft., 5 -gal. can, $\$ 2.00$ ea. $2 \frac{1}{2} \mathrm{ft}$., 5 -gal. can, $\$ 3.00$ ea. $3-3 \frac{1}{2}$ ft., 5 -gal. can, $\$ 3.50$ ea. 4 ft., 5 -gal. can, $\$ 4.00$ ea. $5 \mathrm{ft}$., 5 -gal. can, $\$ 5.00$ ea.

BOTTLE TREE (Sterculia diversifolia). 1-2 ft., pot, $40 \mathrm{c}$ ea., $\$ 3.50$ per 10 . 3-4 ft., 5-gal. can, $75 \mathrm{c}$ ea.

CALIFORNIA LAUREL (Umbellularia). 1-2 ft., pot, $75 \mathrm{c}$ ea., $\$ 6.50$ per $10.2-3 \mathrm{ft}$., 5 -gal. can, $\$ 1.25$ ea.
CALIFORNIA PEPPER TREE (Schinus Molle). 3-4 ft., pot, $50 \mathrm{c}$ ea., $\$ 4.50$ per $10.4-6 \mathrm{ft}$., pot, $75 \mathrm{c}$ ea., $\$ 6.50$ per $10.3-4 \mathrm{ft} ., 1$-gal. can, $50 \mathrm{c}$ ea., $\$ 4.50$ per 10 . From Brazil (S. terebinthifolius). 3-4 ft., 1 -gal. can, $60 \mathrm{c}$ ea., $\$ 5.00$ per 10.

CAROB (ceratonia siliqua). 1-2 ft., pot, $50 \mathrm{c}$ ea. $2-3 \mathrm{ft}$., pot, $75 \mathrm{c}$ ea.

CAMPHOR TREE. 1-2 ft., pot, $50 \mathrm{c}$ ea., $\$ 4.50$ per 10 . 2-3 ft., pot, $75 \mathrm{c}$ ea., $\$ 6.50$ per 10 .

CASSIA GRANDIFLORA. . Large panicles, pink flowers; does well in dry places. 1-2 ft., pot, $50 \mathrm{c}$ ea.

CATALINA I. CHERRY (Prunus integrifolia). $10-16$ in., pot, $20 \mathrm{c}$ ea., $\$ 1.50$ per $10.1-2 \mathrm{ft}$., pot, $25 \mathrm{c}$ ea., $\$ 2.00$ per $10.2-4 \mathrm{ft}$., pot, $40 \mathrm{c}$ ea. 1-3 ft., can, $25 \mathrm{c}$ ea., $\$ 2.00$ per 10 .

CEDAR (Cedrus Atlantica) tall, symmetrical. 2 ft., 5-gal. can, $\$ 2.00$ ea., $\$ 15.00$ per 10 . $2-3$ ft., 5-gal. can, $\$ 2.50$ ea., $\$ 20.00$ per 10 . $3-4$ ft., 5-gal. can, $\$ 3.50$ ea., $\$ 30.00$ per 10 . 4-6 ft., 5- gal. can, $\$ 4.00$ ea., $\$ 35.00$ per 10 .

CEDAR, Deodar. Handsomest Cedar. $3 \mathrm{ft}$, 5 -gal. can, $\$ 2.50$ ea., $\$ 20.00$ per $10.3-4$ ft., 5 - gal. can, $\$ 3.00$ ea., $\$ 25.00$ per 10 . $5 \mathrm{ft}$., 5 -gal. can, $\$ 3.50$ ea.

CEDAR OF LEBANON. Foliage darker; habit more drooping than Atlantica. 2 ft., 5-gal. can, $\$ 2.00$ ea. $2-3$ ft., 5 -gal. can, $\$ 2.50$ ea. $3-4$ ft., 5-gal. can, $\$ 3.50$ ea. 4-6 ft., 5-gal. can, $\$ 4.00$ ea.

CYPRESS (Cupressus) Arizona. Bluish foliage; succeeds well in dry, sunny locations; beautiful and exceedingly useful in California. 1-2 ft., pot, $40 \mathrm{c}$ ea., $\$ 3.50$ per 10 . 2-3 ft., pot, $60 \mathrm{c}$ ea., $\$ 5$ per 10 . 3-4 ft., 5 -gal. can, $\$ 1.25$ ea.

CYPRESS (Benthami). Similar to above. 2-3 ft., pot, $60 \mathrm{c}$ ea., $\$ 5.00$ per 10 .

CYPRESS (Crippsi-aurea). Specimen plants. 5gal. can, $\$ 3.00$ ea.

CYPRESS (erecta viridis). Specimen plants. 5 -gal. can, $\$ 1.50$ ea.

CYPRESS (funebris). Large, spreading habit; foliage light green. $1-2 \mathrm{ft}$., pot, $50 \mathrm{c}$ ea., $\$ 4.00$ per 10. 2-3 ft., pot, $60 \mathrm{c}$ ea., $\$ 5.00$ per 10 .

CYPRESS (glabra). Smooth Cypress. From Arizona, leaves bright blue-green, habit compact. $1-2 \mathrm{ft}$., pot, $60 \mathrm{c}$ ea., $\$ 5.00$ per 10 . $2-3 \mathrm{ft}$., pot, $75 \mathrm{c}$ ea., $\$ 6.00$ per 10 .

CYPRESS (glauca). California; very beautiful. $3-4$ ft., pot, $75 \mathrm{c}$ ea., $\$ 6.50$ per 10 . Specimen plants, 5-gal. can, $\$ 1.00$ ea.

CYPRESS (gracilis). Slender habit; specimen. $4 \mathrm{ft}$., box, $\$ 4.00$ ea.

CYPRESS (lawsoniana-alumi). Compact habit; foliage blue-green; specimen. $2 \mathrm{ft}$., 5 -gal. can, $\$ 2.00$ ea.

CYPRESS (lobbii). Upright grower; specimen. $4 \mathrm{ft}$., 5 -gal. can, $\$ 3.00$ ea.

CYPRESS (lutescens). Delicate foliage; specimen. 5-gal. can, $\$ 1.50$ ea.

CYPRESS (macrocarpa). Monterey Cypress. 6-8 ft., 5-gal can, $\$ 3.00$ ea. Flats, $\$ 3$ per 100 .

CYPRESS. Milford Blue Jacket. Foliage blue. $21 / 2$ ft., 5-gal. can, $\$ 2.50$ ea. 3 ft., 5 -gal can, $\$ 3.00$ per 10 .

CYPRESS (Sempervirens). Italian Cypress. 1-11/2 $\mathrm{ft}$., pot, $50 \mathrm{c}$ ea. $\$ 4.50$ per $10.3-4 \mathrm{ft}$., balled, or 5-gal. can, $\$ 1.00$ ea. $6-8 \mathrm{ft}$., 5-gal. can, $\$ 3.00$ ea.

CYPRESS (Westermanni). Specimen. 2-3 ft., 5 -gal. can, $\$ 2.00$ ea.

EUCALYPTUS. A most useful family, for wood or for ornament. Plants in cans may be set in the ground entire; the can will soon rust away. Write us for information on best varieties for any purpose. 
E. AMPLIFOLIA. Hardy. 1-2 ft., cans, 20c ea., $\$ 1.50$ per 10

E. BOSISTOIANA. Hardy. 1-2 ft., cans, $25 \mathrm{c}$ ea. . CALOPHYLLA. Rather tender. Coast. Large white or pinkish flowers. Ornamental. 1-2 ft., pot, $50 \mathrm{c}$ ea.

E. CITRIODORA. Lemon scented. Rather tender. 1-2 ft., pot, $30 \mathrm{c}$ ea., $\$ 2.50$ per 10 . $2-3$ $\mathrm{ft}$., pot, $40 \mathrm{c}$ ea., $\$ 3.50$ per 10 .

E. CORYNOCALYX. Sugar gum. Hardy. 3-4 ft., 1 -gal. can, $40 \mathrm{c}$ ea.

E. CREBRA. Iron-bark. Narrow leaves. Hardy. $3 \mathrm{ft}$., pot, $25 \mathrm{c}$ ea. Flats $\$ 2.50$ per 100 .

E. DIVERSICOLOR. Tall tree; coast counties. 1-2 ft., can, $25 \mathrm{c}$ ea.

E. ERYTHRONEMA. Hardy. One of the most ornamental; very graceful. 6-12 in., pot, 25 c ea.

E. FICIFOLIA. Tender. Handsome, large red flowers. 1-2 ft., pot, $75 \mathrm{c}$ ea.

E. GLOBULUS. Blue gum. 2-3 $\mathrm{ft}$, pot or can, $25 \mathrm{c}$ ea., $\$ 2.00$ per $10 ; \$ 15.00$ per 100 . $3-4 \mathrm{ft}$. pots, $35 \mathrm{c}$ ea., $\$ 3.00$ per 10 . Flats, $\$ 2.50$ per $100, \$ 20.00$ per 1000 .

E. GLOBULUS COMPACTA. Forms dense round head. 1-2 ft., pot, $40 \mathrm{c}$ ea. $3-4 \mathrm{ft}$, pot, $75 \mathrm{c}$ ea.

E. GONIOCALYX. Tall; hardy. 2-3 ft., can $25 \mathrm{c}$ ea., $\$ 2.00$ per 10 .

E. GUNNII. Moderate grower. Very hardy. $1 \mathrm{ft}$., can, $25 \mathrm{c}$ ea.

E. LEỦCOXYLON-ROSEA. Pink flowers; hardy. 1-2 ft., can, $35 \mathrm{c}$ ea., $\$ 3.00$ per 10 . $2-3 \mathrm{ft}$., can, $40 \mathrm{c}$ ea.

E. LINEARIS. Very graceful, delicate, almost feathery foliage. Hardy. 1-2 ft., pot, $40 \mathrm{c}$ ea., $\$ 3.50$ per 10 .

E. MACULATA. Valuable timber tree, coast counties. $2 \mathrm{ft}$., can, $20 \mathrm{c}$ ea. $3 \mathrm{ft}$., 1 -gal. can, $30 \mathrm{c}$ ea.

E. POLYANTHEMOS. Foliage roundish; beautiful blue-green; profuse bloomer, valuable for honey. Quite hardy. 1-2 ft., 1-gal. can, $25 \mathrm{c}$ ea., $\$ 2.00$ per $10.2-3 \mathrm{ft}$., $40 \mathrm{c}$ ea.

E. POPULIFOLIA. Resembles preceding. 2-3 ft., can, $40 \mathrm{c}$ ea.

E. PULVERULENTA. Blue foliage. Hardy. 1-2 ft., pot, $35 \mathrm{c}$ ea.

E. ROSTRATA. Red Gum. Very hardy. Best for windbreak in Sacramento Valley. 1-2 ft., can, 20 c ea., $\$ 1.50$ per 10 . 2-3 ft., can, 25 c ea. Flats, $\$ 2.50$ per $100, \$ 20.00$ per 1000 .

E. RUDIS. Desert Gum. Leaves roundish. Very hardy. Windbreak. 6-10 in., pot, 15c ea. Flats, $\$ 2.50$ per $100, \$ 20.00$ per 1000 .

E. SIDEROXYLON. Fine leaves; graceful; hardy. $2-3$ ft., can, $35 \mathrm{c}$ ea.

E. SIDEROXÝLON-ROSEA. Like preceding, but with pink flowers. $1-2 \mathrm{ft}$., can, $35 \mathrm{c}$ ea. $\$ 3.00$ per $10 ; 2-3 \mathrm{ft}$., can, $40 \mathrm{c}$ ea.

E. STUARTINANA. Tall, drooping; hardy. 1-2 ft., can, $30 \mathrm{c}$ ea., $\$ 2.50$ per 10 .

E. TERETICORNIS. Resembles Red Gum, but with broader leaves. 1-2 ft., can, $20 \mathrm{c}$ ea., $\$ 1.50$ per $10.2-3 \mathrm{ft}$., can, $30 \mathrm{c}$ ea., $\$ 2.50$ per 10 Flats, $\$ 2.50$ per $100, \$ 20.00$ per 1000 .

E. VIMINALIS. Very tall growing and quite hardy. Succeeds well in interior valley. 2-3 ft., can $30 \mathrm{c}$ ea., $\$ 2.50$ per $10, \$ 20.00$ per 1000 . Flats, $\$ 2.50$ per $100, \$ 20.00$ per 1000 .

EVERGREEN ELM (Ulmus parvifolius). Very rare, beautiful lawn tree; hardy. 7-8 ft., 5gal. can, $\$ 2.50$ ea.

EVERGREEN THORN (Crataegus crenulata) May be grown as a tree, though generally kept in bush form. Berries scarlet. Small plants for hedge. $3-4 \mathrm{ft}$., pot, $60 \mathrm{c}$ each, $\$ 5.00$ per 10. Flats, $\$ 7.50$ per 100 .

EVERGREEN BALD CYPRESS (Taxodium mucronatum). Most beautiful tree, with feath. ery foliage. For lawn center. Very rare. 4-5 ft., 5-gal. can, $\$ 2.50$ ea.

FIR, Douglas (Abies Douglasi). 21/2-4 ft., 5-gal. can, $\$ 2.50$ ea.
FIR, Nordmanns. 1-2 ft., 5-gal. can, $\$ 1.25$ ea. $\$ 10.00$ per 10

HAKEA LAURINA. Leaves long, narrow; flowers vivid crimson. Very handsome tree; fairly hardy; will stand several degrees of frost; drought-resisting. 6-7 ft., 5-gal, can, $\$ 1.25$ ea.

HOLLY LEAVED CHERRY (California). 4-10 in., pot, $20 \mathrm{c}$ ea., $\$ 1.50$ per $10, \$ 12.50$ per 100 $1 \mathrm{ft}$., pot, $30 \mathrm{c}$ ea., $\$ 2.50$ per 10 . 6-12 in., can, $15 \mathrm{c}$ ea., $\$ 1.25$ per $10, \$ 10.00$ per 100 .

INCENSE CEDAR (Libocedrus). Very stately forest tree of the Sierras. Succeeds almost anywhere. $2 \mathrm{ft}$., can, $\$ 1.50$ ea., $2-3 \mathrm{ft}$., can, $\$ 2.00$ ea. 3-4 ft., can, $\$ 2.50$ ea.

JAPAN REDWOOD (Cryptomeria). One of the most ornamental conifers, and very fine as box plant for several years, when it may be planted in the open. Quite hardy. 2-3 ft., 1gal. can, $75 \mathrm{c}$ ea., $\$ 6.50$ per 10 . $3 \mathrm{ft}$., 5 -gal. can, $\$ 1.00$ ea. $3 \frac{1}{2} \mathrm{ft} ., 5$-gal. can, $\$ 1.50$. $7 \mathrm{ft}$., 5 -gal. can, $\$ 3.50$ ea.

JAPAN REDWOOD (C. elegans). Dense, dwarf habit; strikingly ornamental. $8-10$ in., pot, $50 \mathrm{c}$ ea.

JERUSALEM THORN (Parkinsonia). California. Rare, beautiful tree; leaves needle-like; flowers yellow, fragrant, in long racemes. 8-15 ft. pot, $75 \mathrm{c}$ ea.

JAPAN PRIVET (Ligustrum Japonicum). Dark green foliage, white flowers; moderate size lawn tree; hardy specimens, 2-3 ft., pot, $50 \mathrm{c}$ ea. $\$ 4.00$ per 10 . $3-4 \mathrm{ft}$., pot, $60 \mathrm{c}$ ea., $\$ 5.00$ per 10. 3-4 ft., tubs, $\$ 1.50$ to $\$ 2.50$ ea.

JUNIPER, California. Foliage light blue-green. $1-11 / 2 \mathrm{ft}$., pot, $50 \mathrm{c}$ ea.

JUNIPER BERMUDIANA. Stiff bluish-green foliage. 3-4 ft., 5-gal. can, $\$ 1.50$ ea.

JUNIPER, Irish. 6-10 in., balled, $50 \mathrm{c}$ ea. 1-2 ft., balled, $\$ 1.50$ ea. Variegated. $6-12$ in., balled, 50c ea. 1-2 ft., pot, $\$ 1.25$ ea.

LAWSON CYPRESS (Chamaecyparis). Very handsome tree for broad avenues or for parks. $3-4 \mathrm{ft}$., pot, $\$ 1.50$ ea. 4-6 ft., 5-gal. can, $\$ 3$ ea.

LOQUAT. Usually grown as a shrub, but may be trained into a tree with very fine foliage effect. 3-4 ft., 5-gal. can, $\$ 1.50$ ea. 4-5 ft., 5 -gal can, $\$ 2.50$ ea.

MAGNOLIA GRANDIFLORA. Large, glossy leaves, immense, white, fragrant flowers. 1-2 ft., pot, $50 \mathrm{c}$ ea. $2-3 \mathrm{ft}$., pot, $75 \mathrm{c}$ ea. $3-4 \mathrm{ft}$., 5-gal. can, $\$ 1.00$ ea. 1-2 ft., pot, $\$ 1.00$ ea.

MADRONE (Arbutus menziesii). Most characteristic native California tree. 1-2 ft., pot, $\$ 1.00$ ea., $\$ 7.50$ per 10 . $2-3$ ft., pot, $\$ 1.25$ ea., $\$ 10.00$ per 10. $2-3 \mathrm{ft}$., 5-gal can, $\$ 2.00$ ea. 3-4 ft., 5-gal. can, $\$ 3.50$ ea.

MORETON BAY FIG (Ficus Australis). Similar to well-known "Rubber Tree." Useful for house decoration. 2-3 ft., pot, $\$ 1.50$ ea.

MESQUITE (Prosopis juliflora). Grows in desert lands south. Leaves like those of Locust; pods sweet. Thorny. 1-2 ft., pot, $50 \mathrm{c}$ ea., $\$ 4.00$ per 10. 1-2 ft., 1-gal. can, $60 \mathrm{c}$ ea.

MOUNTAIN LILAC (Ceanothus arboreus). Native of Catalina Island; rare. This and the following grow into good sized trees. Flowers in large panicles, deep blue. 1-1 1/2 ft., pot, $75 \mathrm{c}$ ea.

MOUNTAIN LILAC (C. arboreus Hybridum). 6 12 in., pot, $60 \mathrm{c}$ ea., $\$ 5.00$ per 10 .

NUTMEG TREE (Torreya Californica). 1-1 1/2 ft., pot, $\$ 1.00$ ea., $\$ 8.50$ per 10 .

OAK (Quercus ballota). Acorns edible; tree well adapted to California. Introduced by us from Southern Europe. 11/2-2 ft., pot, $50 \mathrm{c}$ ea., $\$ 4.00$ per 10 . $2-2 \frac{1}{2} \mathrm{ft}$., pot, $75 \mathrm{c}$ ea., $\$ 6.50$ per 10 .

OAK (Q. chrysolepis). California Live Oak. 11/2$21 / 2 \mathrm{ft}$., pot, $75 \mathrm{c}$ ea., $\$ 6.00$ per 10 .

OAK (Q. densiflora) Tan Bark. 8-12 in., 40c ea., $\$ 3.50$ per $10.11 / 2 \mathrm{ft}$., pot, $75 \mathrm{c}$ ea. 
OAK (Q. dumosa). Scrub Oak. $2 \mathrm{ft}$, pot, $75 \mathrm{c}$ ea.

OAK (Q. Engelmanni). 11/2 ft., pot, $75 \mathrm{c}$ ea.

OAK (Q. suber). Cork Oak. 21/2-3 ft., pot, 60c ea., $\$ 5.00$ per 10 .

PHOTINIA SERRULATA. Makes a stately tree; foliage large, often crimson; quite hardy. 6-7 ft., 5-gal. can, $\$ 2.50$.

PINE (Pinus alba). Grows well in California. 3 ft., 5-gal. can, $\$ 1.00$ ea.

PINE (P. Austriaca). Tall, handsome pine. 2-3 ft., pot, $75 \mathrm{c}$ ea. $3-4 \mathrm{ft}$., pot, $\$ 1.00$ ea. $3 \mathrm{ft}$, 5-gal. can, $\$ 1.00$ ea. $3-4$ ft., 5-gal. can, $\$ 1.25$ ea. 4-5 ft., 5-gal. can, $\$ 1.50$ ea. $6-8 \mathrm{ft}$., 5gal. can, $\$ 3.50$ ea.

PINE (P. Canariensis). Spreading in habit. Rapid grower; lustrous foliage. 1-2 ft., pot, $50 \mathrm{c}$ ea. 2-3 ft., pot, $75 \mathrm{c}$ ea.

PINE (P. divaricata). Jack Pine. California. 3-4 $\mathrm{ft}$., pot, $75 \mathrm{c}$ ea. $4-6 \mathrm{ft}$., pot, $\$ 1.00$ ea. $6-7 \mathrm{ft}$., 5-gal. can, $\$ 3.00$ to $\$ 3.50$ ea.

PINE (P. Fremontiana). Small, but beautiful pine; California. 2-3 ft., pot, $\$ 1.00$ ea.

PINE (P. insignis-or radiata). Monterey Pine. 2-3 ft., pot or can, $50 \mathrm{c}$ ea, $\$ 4.50$ per $10, \$ 40.00$ per 100. 3-4 ft., pot or can, $75 \mathrm{c}$ ea., $\$ 6.50$ per $10, \$ 50.00$ per 100 . 6-7 ft., 5-gal. can, $\$ 1.50$ ea.

PINE (P. Montana). Dwarf Pine. California. $3 \mathrm{ft}$., 5 -gal. can, $\$ 1.50$ ea.

PINE (P. muricata). California. 6-10 in., pot, $35 \mathrm{c}$ ea.

PINE (P. ponderosa). Yellow Pine. California. $2-3 \mathrm{ft}$., pot or can, $\$ 1.50$ ea.

PINE (P. sylvestris). Scottish Pine. 4-6 ft., 5gal. can, $\$ 1.25$ ea. $7-8$ ft., 5 -gal. can, $\$ 3.00$ to $\$ 3.50$ ea.

PINE (P. tuberculata). California. 8-10 in., pot, $35 \mathrm{c}$ ea.

PITTOSPORUM PHYLLEROIDES. Drooping tree, resembling evergreen weeping willow. Very graceful; new; hardy. 1-2 ft., pot, $50 \mathrm{c}$ ea., $\$ 4.00$ per $10.2-3 \mathrm{ft}$., pot, $75 \mathrm{c}$ ea., $\$ 6.00$ per 10.

PITTOSPORUM EUGENOIDES. Small Tree: often used as a shrub; or for hedges. Foliage light green. 10-12 in., pot, $30 \mathrm{c}$ ea., $\$ 2.50$ per 10. $2-3 \mathrm{ft}$., pot, 50c ea., $\$ 4.00$ per 10 . $10-15$ in., cans, $20 \mathrm{c}$ ea., $\$ 1.50$ per 10 .

PITTOSPORUM NIGRICANS (tenuifolium). Small tree, or used as a shrub, or for hedges; leaves small. $1-2$ ft., pot, $20 \mathrm{c}$ ea., $\$ 1.50$ per 10 . $2-3$ ft., pot, $50 \mathrm{c}$ ea., $\$ 4.00$ per 10 . $10-15$ in., can, $20 \mathrm{c}$ ea., $\$ 1.50$ per 10 .

PITTOSPORUM RHOMBIFOLIUM. Grows to large tree; flowers white. 8-10 in., pot, $35 \mathrm{c}$ ea.

REDWOOD (Sequoia sempervirens). We have a magnificent stock of this justly celebrated tree; all sizes. 3-4 ft., balled, $\$ 1.50$ ea., $\$ 12.50$ per $10, \$ 100.00$ per $100.3-5$ ft., 5-gal. can, $\$ 2.50$ ea., $\$ 20.00$ per 10 . $7-8$ ft., balled, $\$ 5.00$ ea.

RETINOSPORA ERICOIDES. Small, upright grower; foliage light green, almost feathery. $10-12$ in., pot, $50 \mathrm{c}$ ea., $\$ 4.00$ per 10 .

RETINOSPORA PLUMOSUS-AUREA. Foliage golden tinted. $2-3 \mathrm{ft}$., 5 gal. can, $\$ 3.00$ ea.

SHE-OAK (Casuarina equisetifolia). Branches drooping. pruned to a round head, the effect is very pleasing. Hardy. 3-4 $\mathrm{ft}$., pot, $50 \mathrm{c}$ ea., $\$ 4.00$ per $10 ; 5-6 \mathrm{ft}$., pot, $75 \mathrm{c}$ ea., $\$ 6.50$ per 10 .

SHE-OAK (C. stricta). Upright grower. Hardy. 2-3 ft., pot, $50 \mathrm{c}$ ea., $\$ 4.00$ per $10 ; 3-4 \mathrm{ft}$., pot, $60 \mathrm{c}$ ea., $\$ 5.00$ per 10 .

SILK-TASSEL TREE (Garrya elliptica). Calif. Leaves dark green, holly-like; male flrs. in long catkins, often over a foot in length. Small tree. 8-12 in., pot, $50 \mathrm{c}$ ea.; $1-2 \mathrm{ft}$., pot, $75 \mathrm{c}$ ea.
SILKY OAK (Grevillea robusta). 2-3 ft., pot, $50 \mathrm{c}$ ea., $\$ 4.00$ per $10 ; 4-6 \mathrm{ft}$., pot, $75 \mathrm{c}$ ea., $\$ 6.00$ per ten.

SPRUCE (Picea excelsa). Norway Spruce. Symmetrical; strong grower; best for Christmas trees. 2-3 ft., 5 gal. can, $\$ 2.00$ ea.; $3-4 \mathrm{ft}$., 5 gal. can, $\$ 3.00$ ea.; $3-4 \mathrm{ft}$., tubs, $\$ 4.00$ ea.

SPRUCE (P. Mariana). Black Spruce. Small tree. $1 \frac{1}{2} \mathrm{ft}$., pots, $\$ 1.00$ ea.

SPRUCE (P. rubra). Red Spruce. 6-12 in., balled, $60 \mathrm{c}$ ea.

SPRUCE (Abies Menziesii). Sitka Spruce. Tall, slender. $2 \frac{1 / 2}{-3} \mathrm{ft}$., 5 gal. can, $\$ 2.00$ ea.; $3-4 \mathrm{ft}$., 5 gal. can, $\$ 3.00$ ea.

SPRUCE (A. pungens). Colorado Blue Spruce. $1 \frac{1 / 2}{2}-2 \mathrm{ft} ., 5$ gal. can, $\$ 2.50$ ea.

SPRUCE (A. Engelmanni). Tall, Spreading. 2 ft., pot, $60 \mathrm{c}$ ea.

TREE TOBACCO (Nicotiana glanca). Native tree with large, shining leaves and yellow flrs. 3-4 ft., $75 \mathrm{c}$ ea.; $\$ 6.50$ per 10 .

TRISTANIA CONFERTA. Handsome shade tree from New South Wales. Withstands drought. 1-2 ft., pot, 60c ea.

WAX MYRTLE (Myrica California). One of the most beautiful native trees; leaves glossy. 1 ft., pot. $\$ 1.50$ ea.

WESTERN CHINQUAPIN (Castanopsis). California Rare. 6-10 in., pot, $\$ 1.50$ ea.
EW. English. Dark green, spreading. $1-2 \mathrm{ft}$., pot, $\$ 1.00$ ea.

YEW. Irish. Upright. $1 \mathrm{ft}$, pot, $75 \mathrm{c}$ ea.

YEW. Variegated. 2 ft., 5 gal. can, $\$ 2.00$ ea.

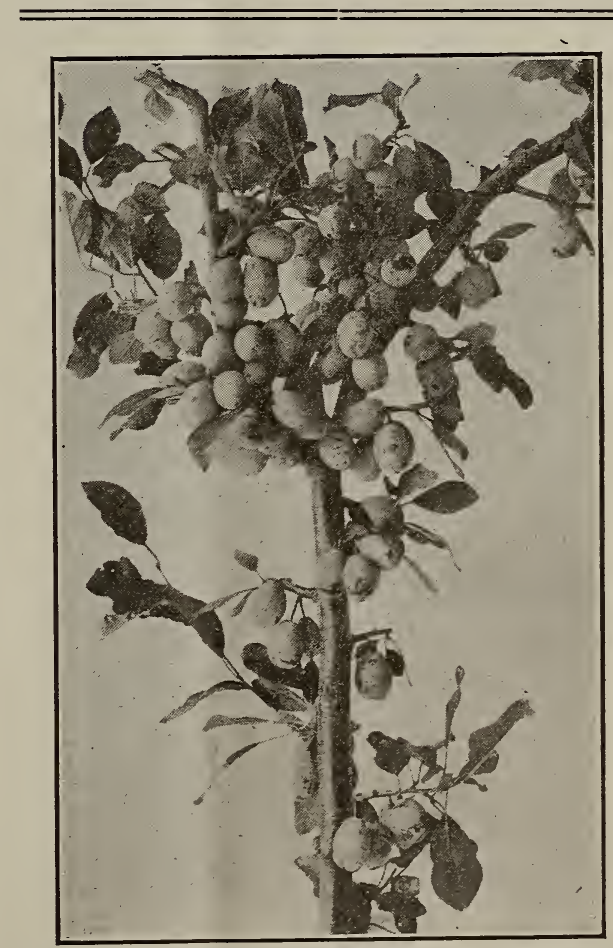

How a "bred up" Coates French Prune will bear on two-year-old wood, with fruit on the spurs along the main limbs 


\section{DECIDUOUS SHRUBS}

ABUTILON. In red, yellow, and white; firs. large, bell-shaped. 1-2 ft., pot, $35 \mathrm{c}$ ea., $\$ 3.00$ per 10 .

ALL-SPICE (Calycanthus occidentalis). Sweet scented shrub; lvs. large; flowers dark red. 2-3 ft., bare root, $50 \mathrm{c}$ ea.

ANGELS' TRUMPET (Datura). Flrs. very large, white. $2-3 \mathrm{ft}$, pot, $50 \mathrm{c}$ ea., $\$ 4.00$ per 10 .

BARBERRY (Thunbergii). Lvs. smaller. Very brilliant coloring. $2-3 \mathrm{ft}$., open ground, $60 \mathrm{c}$ ea., $\$ 5.00$ per 10 .

BARBERRY (Berberis). Common. Flrs. yellow ; fruit crimson; foliage light green. $2-3 \mathrm{ft}$., pot, $40 \mathrm{c}$ ea., $\$ 3.50$ per $10 ; 2-3 \mathrm{ft}$., open ground, $60 \mathrm{c}$ ea.. $\$ 5.00$ per 10 .

BARBERRY. Purple leaved. 2-3 ft., pot, $50 \mathrm{c}$ ea.; 2-3 ft., open ground, $60 \mathrm{c}$ ea., $\$ 5.00$ per 10 .

BIRD OF PARADISE (Caesalpina). Very showy. Red and yellow firs. 1-2 ft., pot, $50 \mathrm{c}$ ea.; $4-6$ ft., open ground, $75 \mathrm{c}$ ea., $\$ 6.50$ per 10 .

BLUEE SPIREA (Caryopteris). $2-3$ ft., 1 gal. can, $50 \mathrm{c}$ ea., $\$ 4.00$ per 10 .

CURRANT (Ribes aureum). Yellow flrs. 1-2 ft., pot, $50 \mathrm{c}$ ea.

CURRANT (R. glutinosum): Calix. Incense Shrub; firs, pink. 1-2 ft., pot, $50 \mathrm{c}$ ea., $\$ 4.00$ per 10 .

CURRANT * (R. malvaceum). Flrs. dull red to purple. California. $5-10$ in., pot, $40 \mathrm{c}$ ea.

DEUTZIA. In white, double and single. Single and double pink, and single deep red. $2-3 \mathrm{ft}$., open ground, $50 \mathrm{c}$ ea., $\$ 4.00$ per 10 ; $3-4$ ft., open ground, $60 \mathrm{c}$ ea., $\$ 5.00$ per 10 .

DOGWOOD (Cornus Californica). 1-2 ft., pot, $50 \mathrm{c}$ ea.. $\$ 4.00$ per 10 .

DOGWOOD (C. Florida). Lvs. and flrs. large; berries red; very handsome shrub. 1-2 ft., pot. $50 \mathrm{c}$ ea., $\$ 4.00$ per 10 .

DOGWOOD (C. Nuttallii). California. Very large flrs. Rare. 6-10 in., pot, $50 \mathrm{c}$ ea.

DUCK PLANT (Colutea). Showy deep red flrs., and large bladder-like pods. $1-2 \mathrm{ft}$, pot, $40 \mathrm{c}$ ea., $\$ 3.50$ per 10 .

DWARF JAPAN CHERRY (Prunus tomentosa). Shrub or bush, covered with small, scarlet, edible fruit. Exceedingly showy. 1-2 ft., 1 gal. can, $60 \mathrm{c}$ ea.; $1-2 \mathrm{ft} ., 5$ gal. can, $\$ 1.00$ ea. GOOSEBERRY (Ribes speciosum). California. Fuchsia flowered gooseberry. Fruit red, bristly. $1-2 \mathrm{ft}$., pot, $50 \mathrm{c}$ ea., $\$ 4.50$ per 10 .

GOOSEBERRY (R. tenuifolium). California. Buffalo berry. Flrs. Yellow; fruit red. 3-5 in., pot, $30 \mathrm{c}$ ea.

GLOBE FLOWER (Kerria). Tall shrub; large double yellow flrs. $2-3 \mathrm{ft}$., pot, $50 \mathrm{c}$ ea., $\$ 4.00$ per $10 ; 3-4 \mathrm{ft}$., pot, $60 \mathrm{c}$ ea., $\$ 5.00$ per 10 .

GLOBE FLOWER. Single flrs, $2-3 \mathrm{ft}$., pot, $60 \mathrm{c}$ ea.

GOLDEN BELL (Forsythia suspensa). 2-3 ft., pot, $40 \mathrm{c}$ ea., $\$ 3.50$ per $10 ; 3-4 \mathrm{ft}$., pot, $60 \mathrm{c}$ ea., $\$ 5.00$ per 10 .

GOLDEN BELL (F. viridissima). Flowers of both are bright yellow, completely covering the drooping branches in early spring. The latter is of more erect habit. 3-4 ft., pot, $60 \mathrm{c}$ ea.

HONEYSUCKLE (Lonicera grandiflora). Upright shrub; flowers scarlet. $3 \mathrm{ft}$., open ground, $50 \mathrm{c}$ ea.

HIBISCUS (Althea). Large shrub, with exceedingly handsome flowers, quite hardy. Single white. Double blue, double red, double purple. Requires water frequently during summer. 3-4 ft., open ground, $60 \mathrm{c}$ ea., $\$ 5.00$ per 10

HIBISCUS MANIHOT. Very large pale yellow flowers with purple eye. 2-3 ft, pot, $60 \mathrm{c}$ ea

HIBISCUS DIVERSIFOLIUS. Tall shrub, flowers very large, yellow, red centre. 3-4 ft., pot, $\$ 1.25$ ea.

HYDRANGEA HORTENSIS. Low shrub. Flowers in immense clusters, white bluish, or pink. $1 \mathrm{ft}$., pot, $40 \mathrm{c}$ ea., $\$ 3.50$ per $10 ; 1-2 \mathrm{ft}$, pot, $60 \mathrm{c}$ ea., $\$ 5.00$ per $10 ; 1-2 \mathrm{ft} .$, tub, $\$ 1.25$ ea.
HYDRANGEA NIGRA. Deep pink. 1 ft., pot, $50 \mathrm{c}$ ea., $\$ 4.50$ per $10 ; 1-2 \mathrm{ft}$., tub, $\$ 1.25$ ea.

HYDRANGEA MOUSSELINE. Deep pink; new. $1 \mathrm{ft}$., pot, $75 \mathrm{c}$ ea.; $1-2 \mathrm{ft} ., \mathrm{tub}, \$ 1.50$ ea.

HYDRANGEA PANICULATA. White, or changing to light pink. $1 \mathrm{ft}$., pot, $40 \mathrm{c}$ ea., $\$ 3.50$ per

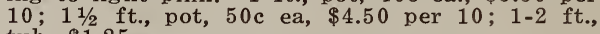
tub, $\$ 1.25$ ea.

JAPAN FLOWERING QUINCE. (Cydonia). 1-2 ft., 5-gal. can, $60 \mathrm{c}$ ea.

LILAC (Syringa vulgaris). Purple. 3-4 ft., can, $50 \mathrm{c}$ ea.

LILAC. Cut-leaf; firs. purple. $3 \mathrm{ft}$., open ground, $50 \mathrm{c}$ ea., $\$ 4.00$ per $10 . ; 3 \mathrm{ft}$., pot, $60 \mathrm{c}$ ea.

LILAC. Flowers lavender. $2 \frac{1}{2} \mathrm{ft}$., pot, $50 \mathrm{c}$ ea. LILAC. Josikaea. Flowers lavender. $1 \mathrm{ft}$., pot, $40 \mathrm{c}$ ea.

LILAC. Flowers white. $2 \mathrm{ft}$., pot, $50 \mathrm{c}$ ea., $\$ 4.00$ per $10 ; 3 \mathrm{ft}$., open ground, $50 \mathrm{c}$ ea.

LILAC. Mad. Lemoine. Large trusses, white flrs. 2-3 ft, pot, $60 \mathrm{c}$ ea.

LILAC. Pres. Grevy. Flowers purplish. 2-3 ft., pot, $60 \mathrm{c}$ ea.

LILÁC. Rubra de Marly. Reddish. 1-2 ft., pot, $60 \mathrm{c}$ ea.

MATILIJA POPPY (Romneya Coulteri). Flowers immense, single white, yellow centre. 1-2 ft., pot, $60 \mathrm{c}$ ea., $\$ 5.00$ per $10 ; 2-3$ ft., tub, $\$ 1.25$ ea.

MOCK ORANGE (Philadelphus). Flowers white 1-2 ft., pot or can, $40 \mathrm{c}$ ea., $\$ 3.00$ per $10 ; 3-4 \mathrm{ft}$., open ground, $60 \mathrm{c}$ ea.

MOCK ORANGE (P. Lewisii) Calif. 2-3 ft., 1 gal. can, $40 \mathrm{c}$ ea., $\$ 3.50$ per 10 .

OSO BERRY (Nuttalia) Calif. Large red and black berries. 1 ft., pot, $\$ 1.00$ ea.

PAW-PAW (Asimina). Ornamental shrub; fruit edible; leaves very large, handsome. 1-2 ft., bare root, $50 \mathrm{c}$ ea.

PEARL BUSH (Exochorda). Large, pure white flrs.; very beautiful spring bloomer. 2-3 ft., bare root, $60 \mathrm{c}$ ea., $\$ 5.50$ per 10 .

POMEGRANATE (Punica). Dbl. red; large. 1-2 ft., 1 gal. can, $40 \mathrm{c}$ ea.

POMEGRANATE. Dbl. white. $2-3 \mathrm{ft.,} 1 \mathrm{gal}$. can, 50 c ea., $\$ 4.00$ per $10 ; 3-4 \mathrm{ft}$, open ground, 60 c ea., $\$ 5.00$ per 10 .

POMEGRANATE. Paper shell. 1-2 ft., pot or can, $40 \mathrm{c}$ ea.

POMEGRANATE. Sweet fruited. 1-2 ft., pot, $40 \mathrm{c}$ ea., $\$ 3.50$ per $10.2 \mathrm{ft}$., open ground, $50 \mathrm{c}$ ea., $\$ 4.00$ per 10 .

POMEGRANATE. Wonderful. Large fruit. 1-2 ft., pot, $50 \mathrm{c}$ ea.

PRIVET (Ligustrum vulgare). Tall; firs. white, very fragrant. $3-4 \mathrm{ft}$., pot, $40 \mathrm{c}$ ea., $\$ 3.00$ per 10.

SALVIA GREGGII. Hard-wooded. Flowers bright crimson; succeeds in hot, dry locations. 1-2 ft., pot, $40 \mathrm{c}$ ea., $\$ 3.50$ per 10 .

SALVIA G. ALBA. Same, with white firs. 1-2 ft., pot, $40 \mathrm{c}$ ea., $\$ 3.50$ per 10 .

SMOKE TREE (Rhus cotinus). Large bush; leaves shiny; flrs. pinkish. $3-4 \mathrm{ft} .$, can, $60 \mathrm{c}$ ea., $\$ 5.00$ per 10 .

SNOW-DROP TREE (Halesia). Large shrub, with drooping white flrs. 2-3 ft., bare root, $75 \mathrm{c}$ ea.

STRYAX CALIFORNIA. Related to above. Very rare. Small plants. Pot, $\$ 1.50$ ea.

SPIREA ALBA-FLORA. Flowers white to pink. 1-2 ft., pot, $40 \mathrm{c}$ ea.

SPIREA. Anthony Waterer. Flowers crimson. 1-2 ft., pot, $40 \mathrm{c}$ ea.

SPIREA ARGENTEA. Flowers small, white. 4 ft., bare root, $50 \mathrm{c}$ ea.

SPIREA BUMALDA. Flowers white to pink. 1-2 ft., pot, $40 \mathrm{c}$ ea., $\$ 3.50$ per 10 . 
SPIREA CANESCENS. Tall; flrs, white. $4 \mathrm{ft}$., bare root, $50 \mathrm{c}$ ea., $\$ 4.00$ per 10 .

SPIREA DOUGLASII. Tall; flrs., pink. 2-3 ft., pot or can, $50 \mathrm{c}$ ea., $\$ 4.00$ per 10 .

SPIREA REEVSIANA. Leaves glaucous; flrs. white. 2-3 ft., pot, $50 \mathrm{c}$ ea., $\$ 4.00$ per 10 .

SPIREA VAN HOUTTII. Bridal Wreath. 1-2 ft. 1 gal. can, $40 \mathrm{c}$ ea., $\$ 3.50$ per 10 .

TAMARIX AESTIVALIS. Tall; flrs. pink spring. $4 \mathrm{ft}$., bare root, $60 \mathrm{c}$ ea., $\$ 5.00$ per $10 ; 3-4 \mathrm{ft}$., 5 gal. can, $60 \mathrm{c}$ ea.

TAMARIX PARVIFLORA. Late summer. 3-4 ft., 5 gal. can, $60 \mathrm{c}$ ea.

THORN (crataegus coccinea). Scarlet Thorn. $2-3 \mathrm{ft}$., bare root, $60 \mathrm{c}$ ea., $\$ 5.00$ per 10 . $3-4 \mathrm{ft}$., bare root, $75 \mathrm{c}$ ea., $\$ 6.00$ per 10 .

THORN (C. crus-galli). Large leaves and berries; beautiful autumn colors. $2-3$ ft., bare root, $60 \mathrm{c}$ ea., $\$ 5.00$ per $10 ; 3-4 \mathrm{ft}$., bare root, $75 \mathrm{c}$ ea., $\$ 6.00$ per 10 . These should be much more largely planted in California; they may be grown into quite large trees, or keptt lo:v.
TREE MALLOW (Malva assurgentifolia). Large shrub or tree; flrs. large, crimson; grows well in dry locations; often used as a windbreak for vegetable gardens near the coast. 2-3 ft., pot, $40 \mathrm{c}$ ea., $\$ 3.50$ per 10 .

TREE POPPY. Dendromecon. California. Large shrub, flowers yellow. The only hard-wooded poppy. Very rare. 1-2 ft., pot, $\$ 1.50$ ea.

TURK'S CAP (Achania). Flowers scarlet, shaped like a cap or bell; showy. 3-4 ft., pot., $40 \mathrm{c}$ ea.

WAX-BERRY (Symphoricarpus) Calif. Fine shrub for massing under oaks, as a cover. Berries either red or white. $1 \frac{1 / 2-2}{\mathrm{ft}}$., pot, $35 \mathrm{c}$ ea., $\$ 3.00$ per $10, \$ 25.00$ per 100 .

WEIGELA (Diervilla). Well-known shrub with handsome flrs. in pink, deep crimson, or rose. $2-3 \mathrm{ft}$., pot, $50 \mathrm{c}$ ea., $\$ 4.00$ per 10 .

VIBURNUM (Snowball). 1-2 ft., pot, $40 \mathrm{c}$ ea.

V. PLICATUM (Japanese Snowball). 1-2 ft., pot, $50 \mathrm{c}$ ea.

\section{EVERGREEN SHRUBS}

ABELIA RUPESTRIS. Mexican shrub, almost everblooming; firs. white or light pink; hardy. $1-2$ ft., pot, $40 \mathrm{c}$ ea., $\$ 3.50$ per 10 .

ARDISIA CRENULATÁ. Shrub with long wavy leaves; drooping clusters coral flrs. $-6-8$ in., pot,

$35 \mathrm{c}$ ea.
AUCUBA. Handsome pot plant, or small shrub; likes partial shade. Lvs. large, spotted or marked with gold. In large pots, $75 \mathrm{c}$ ea; $\$ 6.50$ per 10.

AUCUBA VIRIDIS. Lvs, dark green, $75 \mathrm{c}$ ea.

BREATH OF HEAVEN (Diosma). Small shrub; leaves needle-like; flrs. white. Whole plant very fragrant. $1-2 \mathrm{ft}$., pot, $50 \mathrm{c}$ ea., $\$ 4.00$ per $10 ; 2-3 \mathrm{ft}$., pot, $75 \mathrm{c}$ ea.

BARBERRY (Berberis aquifolium-or Mahonia). Oregon Grape. Fine stock. 1-2 ft., balled, $75 \mathrm{c}$ ea., $\$ 7.00$ per $10 ; 1-2$ ft., 1 gal. can, $60 \mathrm{c}$ ea., $\$ 5.50$ per $10 ; 2-3 \mathrm{ft} ., 5$ gal. can, $\$ 1.00$ ea.,

BARBERRY ( $\dot{B}$. Californica). The most beautiful native shrub; leaves holly-like, changing in color; flrs. yellow; fruit deep blue. 6-10 in., pot, $60 \mathrm{c}$ ea., $\$ 5.50$ per $10 ; 10-12$ in., pot, 75 c ea.; $1-2$ ft., pot, $\$ 1.00$ ea.

BARBERRY (B. darwinii). Another most satisfactory garden shrub. Leaves small, shiny; flrs. numerous, yellow; fruit deep blue. 6-12 in., pot, $40 \mathrm{c}$ ea., $\$ 3.50$ per $10 ; 1-2 \mathrm{ft}$., pot, 60 c ea., $\$ 5.00$ per 10 .

BARBERRY (B. elegantissima). This, and three following, are among the more recent introductions from South America. They are very desirable and well suited to California. Mostly used in low shrubbery effect. 1-2 ft., pot, $60 \mathrm{c}$ ea., $\$ 5.00$ per 10 .

BARBERRY (B. Knightii). Leaves larger; very glossy, dark green. 1-2 ft., pot, $60 \mathrm{c}$ ea.

BARBERRY (B. stenophylla). Graceful, arching branches. $1-21 / 2 \mathrm{ft}$., pot, $50 \mathrm{c}$ ea., $\$ 4.00$ per 10 ; $2-3 \mathrm{ft}$., pot, $75 \mathrm{c}$ ea., $\$ 6.50$ per 10 .

BARBERRY (B. Wilsonae). Very elegant, with beautiful colors in stem, leaves, flrs., and fruit. $1-2$ ft., pot, $75 \mathrm{c}$ ea., $\$ 6.50$ per $10 ; 2-3 \mathrm{ft}$., pot, $85 \mathrm{c}$ ea., $\$ 7.50$ per 10 .

BOTTLE BRÚSH (Callistemon). Flowers crimson. 3-4 ft., 1 gal. can, $60 \mathrm{c}$ ea., $\$ 5.00$ per 10 . BOX (Buxus)." De Mahan. 1-2 ft., open ground. $40 \mathrm{c}$ ea., $\$ 3.00$ per $10, \$ 25.00$ per 100

BOX. Dwarf, for edging. 8 in., open ground, $30 \mathrm{c}$ ea., $\$ 2.50$ per $10 ; 2-3$ in, open ground, $\$ 8.50$ per 100 .

BOX. Elegantissima. Foliage silvery. 8-15 in., open ground, $40 \mathrm{c}$ ea., $\$ 3.00$ per $10, \$ 20.00$ per $100 ; 6-8$ in., open ground, $25 \mathrm{c}$ ea., $\$ 2.00$ per $10, \$ 15.00$ per 100
BOX GOLDEN. 6-8 in., open ground, $25 \mathrm{c}$ ea., $\$ 2.00$ per $10, \$ 15.00$ per 100 .

BOX. Handsworth. Vigorous. 2-2 $1 / 2$ ft., open ground, $50 \mathrm{c}$ ea., $\$ 4.00$ per $10, \$ 30.00$ per 100 . BOX. Microphylla. Low growing. $1 \mathrm{ft}$., open ground, $40 \mathrm{c}$ ea., $\$ 3.00$ per $10, \$ 20.00$ per 100 .

BOX MYRTIFOLIA. Drooping. 1-11/2 ft., open ground, $40 \mathrm{c}$ ea., $\$ 3.00$ per $10, \$ 20.00$ per 100 . $\mathrm{ft}$., open ground, $40 \mathrm{c}$ ea., $\$ 3.00$ per $10, \$ 20.00$ per 100 .

BOX SEMPERVIRENS. Vigorous spreading. $1-1 \frac{1 / 2}{\mathrm{ft}}$., open ground, $40 \mathrm{c}$ ea., $\$ 3.00$ per 10 , $\$ 20.00$ per 100 .

BOX. Trained, in tubs or can. 2-3 ft., tub, $\$ 2.50$ ea.

BROOM (Cytisus, Genista). Andreana. Flowers deep crimson and yellow. 6-12 in., pot, $40 \mathrm{c}$ ea. BROOM (G. Canariensis). Good foliage; branching; profuse yellow flrs. $3-4 \mathrm{ft}$., pot, $40 \mathrm{c}$ ea., $\$ 3.50$ per $10 ; 4-6 \mathrm{ft}$., pot, $75 \mathrm{c}$ ea.

BROOM (C. hispanica) Spanish. Best of the family. 2-3 ft., pot or large can, $50 \mathrm{c}$ ea., $\$ 4.00$ per $10 ; 3-4 \mathrm{ft}$, pot or large can, $60 \mathrm{c}$ ea., $\$ 5.00$ per 10 .

BROOM (C. monosperma). Slender, drooping; Als. white. New. 1-2 ft., pot, $75 \mathrm{c}$ ea.

BROOM (C. monosperma pendula). Quite weeping habit. Rare. $1 \mathrm{ft}$., pot, $75 \mathrm{c}$ ea.

BROOM (C. nigricans). Small shrub. 1-2 ft., pot, $40 \mathrm{c}$ ea.

BROOM (C. racemosus). Flowers in large upright racemes. 1-2 ft., pot, $40 \mathrm{c}$ ea.

BUTTERFLY BUSH (Buddleia davidii). Large shrub; globular panicles, light purple flrs. $2-3$ ft., pot, $50 \mathrm{c}$ ea., $\$ 4.00$ per $10 ; 3-4 \mathrm{ft}$., pot, $60 \mathrm{c}$ ea., $\$ 5.00$ per 10 .

BUDDLEIA GLOBOSA. Roundish heads, yellow flrs. Hardy. 2-3 ft., pot, $50 \mathrm{c}$ ea.

CAMELLIA JAPONICA. Camellias are very scarce. pot, $\$ 1.25-\$ 3.00$ per 10 .

CANTUA BUXIFOLIA. Small shrub; firs. funnelshaped, red. 1-2 ft., pot, $40 \mathrm{c}$ ea.

CASCARA. Coffee Berry (Rhamnus) Calif. Fine shrub; grows well in dry places. 1-2 ft., pot, $60 \mathrm{c}$ ea., $\$ 5.00$ per 10.

COFFEE BERRY (Rhamnus ilicifolia) Calif. Rare. Berries red. $1 \mathrm{ft}$., pot, $\$ 1.50$ ea.

CASSIA ARTEMESOIDES. Roundish shrub. Leaves blue-green, feathery; flrs. yellow. 6-12 in., pot, $35 \mathrm{c}$ ea.

CHAPARRAL (Adenostoma) Calif. Heath-like shrub; flrs. white, terminal racemes. 6-12 in., pot, $50 \mathrm{c}$ ea. 
CHORIZEMA VARIUM. Small shrub, covered with bright orange and crimson pea-shaped flrs., leaves dark green, holly-shaped. Rare. 6-12 in., pot, $50 \mathrm{c}$ ea.

CHRISTMAS BERRY (Heteromeles). Fine stock. $1-2$ ft., pot, $40 \mathrm{c}$ ea., $\$ 3.00$ per $10, \$ 25.00$ per $100 ; 2-3 \mathrm{ft}$., pot, $60 \mathrm{c}$ ea., $\$ 5.00$ per $10 ; \$ 40.00$ per 100

CORAL PLANT (Cestrum). Large leaves; flrs. orange-red, tubular shaped. $3 \mathrm{ft}$. pot, $40 \mathrm{c}$ ea.

COPROSMA BAUERII. Very shining leaves. Makes a large shrub. 2-3 ft., pot, $60 \mathrm{c}$ ea., $\$ 5.50$ per $100 ; 3-4 \mathrm{ft}$., pot, $75 \mathrm{c}$ ea.

CORONILLA EMERUS. Shrub or half-climber; leaves light green; yellow, pea-shaped flrs. all summer. Hardy. 2-3 ft., pot, $50 \mathrm{c}$ ea., $\$ 4.00$ per 10.

CORONILLA GLAUCA. Smaller shrub, leaves blue-green. $2-3$ ft., pot, $50 \mathrm{c}$ ea.

CORONILLA GLAUCA VARIEGATA. Foliage golden. $1 \mathrm{ft}$., pot, $50 \mathrm{c}$ ea.

COTONEASTER. In California most species are practically evergreen. Their bright berries, and adaptability make them among the most useful plants for large or small planting.

C. ANGUSTIFOLIA . Spreading. Berries orange. 1-2 ft., pot, $50 \mathrm{c}$ ea., $\$ 4.00$ per $10 ; 2-3 \mathrm{ft}$., pot, $60 \mathrm{c}$ ea., $\$ 5.00$ per 10

C. FRANCHETTI. Much branched. Berries reddish. $1-2$ ft., pot, $50 \mathrm{c}$ ea., $\$ 4.00$ per $10 ; 2-3$ ft., pot, $60 \mathrm{c}$ ea., $\$ 5.00$ per $10 ; 3-4 \mathrm{ft}$., pot, $75 \mathrm{c}$ ea.

C. FRIGIDA. Tall shrub. Berries scarlet. $3 \mathrm{ft}$., pot, $75 \mathrm{c}$ ea.

C. HÓRIZONTALIS. Procumbent. Berries scarlet. $1-1 \frac{1 / 2}{\mathrm{ft} .,} 4$-in. pot, $60 \mathrm{c}$ ea., $\$ 5.00$ per 10 ; $1-2 \mathrm{ft}$., 5in. pot, $75 \mathrm{c}$ ea., $\$ 6.50$ per $10 ; 2-3 \mathrm{ft}$., 6 in. pot, $\$ 1.00$ ea., $\$ 8.50$ per 10 .

C. MICROPHYLLA. Low, almost prostrate; rich green foliage; crimson berries. 1-2 ft., pot, $60 \mathrm{c}$ ea., $\$ 5.00$ per $10 ; 2-3 \mathrm{ft}$., pot, $75 \mathrm{c}$ ea., $\$ 6.50$ per 10 .

C. PANNOSA. Tall, slender. Berries scarlet. 1-2 ft., pot, $50 \mathrm{c}$ ea., $\$ 4.50$ per $10 ; 2-3 \mathrm{ft}$., pot, $75 \mathrm{c}$ ea., $\$ 6.50$ per $10 ; 3-4 \mathrm{ft}$., pot, $\$ 1.00$ ea., $\$ 8.50$ per $10 ; 4-6$ ft., 5 gal. can, $\$ 2.50$ ea.

C. SIMONSI, Spreading. Red berries. $2-3 \mathrm{ft}$., pot, $75 \mathrm{c}$ ea.

C. THYMIFOLIA. Trailing; dense growing; leaves very small. Red berries. New. 6-12 in., pot, $75 \mathrm{c}$ ea., $\$ 6.50$ per 10 .

CRAPE MYRTLE (Lagerstroemia). Large shrub, with pink or crimson feathery flrs. all summer. Beautiful. 8-12 in., pot, $35 \mathrm{c}$ ea.; 1-2 $\mathrm{ft}$., pot, $50 \mathrm{c}$ ea.; $2-3 \mathrm{ft}$, pot, $75 \mathrm{c}$ ea.

CUPHEA HYSSOPIFOLIA. Small shrub, covered with small tubular lilac flrs. $8-12$ in., pot, $35 \mathrm{c}$ ea.

DAPHNE ODORATA. Flowers pink or white. Very scarce. 1-2 ft., pot, $\$ 1.25-\$ 2.00$ ea

EUONYMUS JAPONICÄ. Úseful shrubs, liking sunlight, and succeeding in dry places. Foliage dark green. $2 \mathrm{ft}$., $35 \mathrm{c}$ ea.; $2-3 \mathrm{ft}$., balled, $\$ 1.00$

ea.

E. JAP. AUREA. Golden foliage. $1 \mathrm{ft}$, pot, $30 \mathrm{c}$ ea., $\$ 2.50$ per $10 ; 1-2 \mathrm{ft} .$, balled, $75 \mathrm{c}$ ea.

E. VARIEGATA. Leaves mottled. 2-3 ft., pot, $50 \mathrm{c}$ ea.

E. DUC D'ANJOU. Leaves marked with gold. 1-2 ft., pot, $40 \mathrm{c}$ ea., $\$ 3.50$ per $10 ; 2 \mathrm{ft}$., balled, $\$ 1.25$ ea., $\$ 10.00$ per 10 .

E. PULCHELLUS. Dwarf; upright; small dark green leaves; fine for hedge. $10-15$ in., pot, $35 \mathrm{c}$ ea., $\$ 3.00$ per $10, \$ 25.00$ per 100 .

E. RADICANS. Trailing. Leaves dark green. 1-2 ft., can. 35c ea., $\$ 3.00$ per 10 .

E. RAD. VARIEGATA. As above, but silvery leaves. 1-2 ft., pot, $40 \mathrm{c}$ ea., $\$ 3.50$ per 10 .

E. SIEBOLDII. Low, spreading; leaves turning red in fall. 2-3 ft., pot, 65c ea., $\$ 5.50$ per 10 .

E. SILVER-LEAVED. 10-15 in., pot, 35c ea. $\$ 3.00$ per $10 ; 11 / 2-2$ ft., balled, $\$ 1.00$ ea., $\$ 9.50$ per $10 ; 21 / 2$ ft., balled, $\$ 1.25$ ea., $\$ 11.50$ per 10 .

ELEAGNUS PUNGENS. Spreading; leaves shining, silvery; red flrs. and fruit. 1-2 ft., pot $50 \mathrm{c}$ ea., $\$ 4.50$ per $10 ; 2-3 \mathrm{ft}$, pot, $75 \mathrm{c}$ ea.; $3-4$ ft., pot, $\$ 1.00$ ea.
ENCELIA CALIFORNICA. Native shrub, with large yellow composite flowers. Rare. pot, $75 \mathrm{c}$ ea.

ESCALLONIA ALBA. Foliage dark, glossy. White flrs. in terminal panicles. Succeeds in sunny, dry location. Hardy. 1-2 ft., 1 gal. can, $50 \mathrm{c}$ ea., $\$ 4.50$ per $10 ; 2-3 \mathrm{ft}$., pot, $75 \mathrm{c}$ ea., $\$ 6.50$ per 10 .

E. EXONIENSIS. Flowers white. New. 8-12 in., pot, $40 \mathrm{c}$ ea., $\$ 3.50$ per $10 ; 1-2 \mathrm{ft} ., 1 \mathrm{gal}$. can, $65 \mathrm{c}$ ea.

E. INGRAMMI. Flowers crimson. New. 2-3 ft. $75 \mathrm{c}$ ea., $\$ 6.50$ per 10 .

E. MACRANTHA. Flowers red. New. 1-2 ft. 1 gal. can, 50c ea., $\$ 4.50$ per 10 .

EUGENIA MYRTIFOLIA. Australian bush cherry. Tall shrub or small tree, with bright myrtlelike leaves, and small red fruit. 2-3 ft., $75 \mathrm{c}$ ea.; $\$ 6.50$ per 10 . 3-4 ft., $\$ 1.00$ ea.

EUGENIA HOOKERIANA. Variety of above $10-15$ in., $50 \mathrm{c}$ ea.

FABIANA IMBRICATA. Heath-like shrub. Profuse bloomer; white. $2-3 \mathrm{ft} ., 1$ gal. can, $40 \mathrm{c}$ ea;

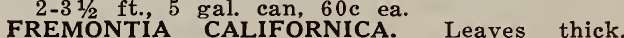
bronze undersides. Flowers large, rich yellow, waxy. One of the handsomest native shrubs of the mountains. Succeeds any where in well-drained soil, if not watered too much. Rare. 6-10 in., pot, $\$ 1.00$ ea.; $10-16$ in., pot, $\$ 1.50$ ea.

FEIJOA SELLOWIANA. Ornamental shrub bearing a highly esteemed fruit. Hardy. 6-12 in., pot, $40 \mathrm{c}$ ea.

GOLDEN DEW-DROP (Duranta). Leaves large, flrs. lilac, with yellow calyx; rather tender, except on the coast. 1-2 ft., pot, $50 \mathrm{c}$ ea.

GORSE (Ulex Europeus). Leaves spiny; flrs. yellow, borne almost all the year. 1-2 ft., pot, $40 \mathrm{c}$ ea., $\$ 3.50$ per 10

GREVILLEA THELIMANNIANA. Leaves finely pinnate; flrs. deep red. Succeeds on the coast. $6-8$ in., pot, $35 \mathrm{c}$ ea.

GROUNDSEL' TREE (Baccharis pilularis-Calif.) Shrub with white flirs. and downy seed; very ornamental. 2-3 ft., pot, $60 \mathrm{c}$ ea., $\$ 5.00$ per 10 . GUAVA. Lemon color. 6-10 in., pot, $40 \mathrm{c}$ ea.

GUAVA. Strawberry. 1-2 ft., pot, 50c ea.; 2-3 ft., pot, $75 \mathrm{c}$ ea.

HAKEA. Ornamental drought-resisting Australian shrubs.

HEATH (Erica carnea). Spring flowering; pink. In 5 -in. pots, large plants, $60 \mathrm{c}$ ea; $\$ 5.00$ per 10 . 6 -in. pots, $75 \mathrm{c}$ ea; $\$ 6.50$ per 10 .

HEATH (E. Cotonoidesveitchii). Spring flowering; white. 8-12 in., $60 \mathrm{c}$ ea.; $\$ 5.00$ per 10 . $1-2 \mathrm{ft} ., 75 \mathrm{c}$ ea.; $\$ 6.50$ per 10

HEATH (E. Felix Fauré). Flowers large, deep pink; blooms almost continuously; new. 8-12 in., $75 \mathrm{c}$ ea. $12-18$ in., $\$ 1.00$ ea.

HEATH (E. Mediterranea). Flowers pink; winter. HEATH (E. melanthera). Flrs. lt. pink; winter.

HEATH (E. vagans). Flrs. pale purple; grows large, spreading; spring.

HEATH (E. vagans rubra). Low-growing; purplish ; spring.

H. ELLIPTICA. Erect; leaves oval; flrs. white. 6-10 in., pot, $40 \mathrm{c}$ ea.

H. SALIGNA. Leaves long, narrow; flrs. white. 1-2 ft., pot, $50 \mathrm{c}$ ea.; $2-3 \mathrm{ft} ., 1$ gal. can, $\$ 1.00$

H. SUAVEOLENS. Leaves rigid, spine-like flrs. white, fragrant. 3-4 ft., 1 gal. can, $\$ 1.00$ ea.

HOLLY (Ilex). Grows best near the coast. 2 ft., pot, $50 \mathrm{c}$ ea.

HOLLY. Japanese (Osmanthus). Holly-like leaves. Flowers white, fragrant. 1-2 ft., pot, $60 \mathrm{c}$ ea., $\$ 5.00$ per $10 ; 2-3$ ft., can, $\$ 1.00$ ea.; $3-4$ ft., 5 gal. can, $\$ 1.25$ ea.

HONEYSUCKLE LONICERA NITIDA. Leaves minute, very dense; flrs. white; berries purple. Very ornamental. New. 1-2 ft., pot, $60 \mathrm{c}$ ea., $\$ 5.00$ per 10.

HUCKLEBERRY (Vaccinium ovatum). 6-10 in. pot $75 \mathrm{c}$ ea. 
HYPERICUM. Dwarf. Foliage large, shining; flrs. large, handsome, yellow. This variety unsurpassed as a ground cover, especially in partial shade. 1-2 ft., can, $35 \mathrm{c}$ ea., $\$ 3.00$ per $10, \$ 20.00$ per $100 ; 2-3 \mathrm{ft}$., can, $50 \mathrm{c}$ ea., $\$ 4.50$ per $10 ; 8-12$ in., can, 20 c ea., $\$ 1.50$ per 10 , $\$ 10.00$ per 100 .

H. MOSERIANUM. Similar to above, but taller growing. 2-3 ft., can, $50 \mathrm{c}$ ea., $\$ 4.00$ per 10 .

H. MOS. TRICOLÓR. Foliage variegated. 1-2 ft., can, $40 \mathrm{c}$ ea., $\$ 3.50$ per 10 .

H. NEPALENSE. 'Tall growing; flrs. smaller. $2-3$ ft., can, $60 \mathrm{c}$ ea., $\$ 5.00$ per 10 .

JUNIPER. Trailing (Juniperus sabina). 1-2 ft., pot, $\$ 1.50$ ea.; 6-10 in., pot, $75 \mathrm{c}$ ea.

JUNIPER. Variegated. Foliage edged silver. 1-2 ft., pot, $\$ 1.50$ ea.

LAUREL. English (Laurus cerasus). Leaves very large, shiny, rich green. Hardy. Extra size, bushy. 2-3 ft., 5 gal. can, $\$ 1.50$ ea.; $2-3$ ft., balled, $\$ 3.00$ ea.

LAUREL. Portugal (L. lusitanica). Leaves long. flrs. white, in drooping racemes. $11 / 2-2 \mathrm{ft} ., 5$ gal. can, $\$ 1.50$ ea.; $1-2$ ft., pot, $75 \mathrm{c}$ ea.

LAURUSTINUS. Compact, free blooming shrub, a favorite everywhere. 1-2 ft., pot, $50 \mathrm{c}$ ea., $\$ 4.50$ per 10 .

LAURUSTINUS VARIEGATA. Same, with variegated foliage. 1-2 ft., pot, $50 \mathrm{c}$ ea.

LEMON. Ponderosa. Ornamental shrub; bearing large lemons of fair quality. 2-3 ft., pot, $\$ 1.00$ ea.

LION'S TAIL (Leonotis). Half-woody shrub; flrs. showy, orange-red. $2-3 \mathrm{ft}$., pot, $40 \mathrm{c}$ ea.

MALLOW (Malvastrum splendidum, California). Leaves downy, white; flrs. in spikes, large, pink, fragiant; very handsome. $50 \mathrm{c}$ ea.

MANZANITA (Arctostaphyllos pungens). 8-12 in., pot, $75 \mathrm{c}$ ea.

MANZANITA (A'. uva-ursi). Trailing. 8-12 in. pot, $75 \mathrm{c}$ ea.

\section{locations.}

M. DECUSSATA. Tall, flrs, white or pinkish. 2-3

M. ERICIFOLIA. Very fine, heath-like foliage. Flowers yellowish. 2-3 ft., pot, $50 \mathrm{c}$ ea., $\$ 4.00$ per $10 ; 4-5 \mathrm{ft}$., pot, $75 \mathrm{c}$ ea.

MIMULUS GLUTINOSA. Calif. Flowers yellow. Grows in dry places. 2-3 ft., pot, $50 \mathrm{c}$ ea.

MOUNTAIN LILAC (Ceanothus). Calif. With the exception of $\mathrm{C}$. arboreus and $\mathrm{C}$. arboreus hybridum, the mountain Lilacs are shrubby.

MT. LILAC (C. divaricatus). Blue to white. 1-2 ft., pot, $40 \mathrm{c}$ ea., $\$ 3.50$ per $10 ; 3-4 \mathrm{ft}$., pot, $60 \mathrm{c}$ ea., $\$ 5.00$ per 10 .

MT. LILAC (C. incanus). Blue. 1-2 ft., pot, $50 \mathrm{c}$ ea.

MT. LILAC (C. interregimus). Lt. Blue. 2-3 ft., pot, $50 \mathrm{c}$ ea.

MT. LILAC (C. megacarpa). White. $1 \frac{11 / 2-21 / 2}{2}$ ft., pot, $50 \mathrm{c}$ ea.

MT. LILAC (C. spinosus). Leaves glaucous firs. sky-blue; one of the most beautiful. 2-3 ft., pot, $50 \mathrm{c}$ ea., $\$ 4.00$ per $10 ; 3-4 \mathrm{ft}$., pot, $60 \mathrm{c}$ ea., $\$ 5.50$ per 10 .

MT. LILAC (C. thyrsiflorus). Foliage rich, glossy green; firs. deep blue. 1-2 ft., pot, $40 \mathrm{c}$ ea., $\$ 3.50$ per $10 ; 2-3 \mathrm{ft}$., pot, $50 \mathrm{c}$ ea., $\$ 4.50$ per $10 ; 3-4 \mathrm{ft} ., 75 \mathrm{c}$ ea., $\$ 6.00$ per 10 .

MT. LILAC (C. verrucosus). Flowers white. 2-3 ft., pot, $50 \mathrm{c}$ ea.

MT. LILAC (Ceanothus Hybridum). Gloire de Versailles. Flowers blue. 1-2 ft., pot, $50 \mathrm{c}$ ea.

MT. LILAC (C. Hybridum). Marie Simon. Flowers pink. 6 in., pot, $50 \mathrm{c}$ ea.

MEXICAN ORANGE (Choisya ternata). Leaves shining; broad panicles, fragrant white flrs. Hardy. $1 \mathrm{ft}$, pot, $50 \mathrm{c}$ ea.

MOCK ORANGE' (Philadelphus Mexicanus). Similar to other species, but evergreen in most parts of California. 1-2 ft., pot, $50 \mathrm{c}$ ea.

MYRTLE (Myrtus communis). Small, bright green leaves, clusters of white flrs. Very pretty as individual shrub near house, or as a hedge. 6-12 in., pot, $35 \mathrm{c}$ ea., $\$ 3.00$ per $10, \$ 25.00$ per 100 .
MYRTLE (M. microphylla). Similar, leaves smaller. 6-12 in., pot, $35 \mathrm{c}$ ea., $\$ 3.00$ per 10 .

NANDINA DOMESTICA. Japanese shrub succeeding well in most parts of California. Grows to about 4 or $5 \mathrm{ft}$., increasing in size by new stalks from the base. Foliage graceful, often changing to deep red. Flowers white in long terminal upright panicles. Berries red, sometimes white. Hardy. Especially recommended. Does well in partial shade, and requires water in the summer. 1-2 ft., pot, $60 \mathrm{c}$ ea., $\$ 5.00$ per $10 ; 2-2 \frac{1}{2} \mathrm{ft}$, tub, $\$ 1.50$ ea.

NIEREMBERGIA. Small shrub or pot plant, covered with single dark violet flrs. Hardy. Very attractive. 1-2 ft., pot; $35 \mathrm{c}$ ea.

OLEANDER (Nerium). Peculiarly adapted to California conditions, thriving best in sunny climate. $1-2$ ft., pot, $50 \mathrm{c}$ ea., $\$ 4.50$ per 10 , $\$ 40.00$ per $100 ; 2-3 \mathrm{ft}$., pot, $75 \mathrm{c}$ ea., $\$ 6.50$ per $10, \$ 55.00$ per 100 ; trained as standards. $3-4$ ft., balled, $\$ 1.00$ ea., $\$ 9.00$ per 10 .

OLEANDER. Double crimson.

OLEANDER. Double pink.

OLEANDER. Double rose.

OLEANDER. Double salmon-pink.

OLEANDER. Double white.

OLEANDER. Single crimson.

OLEANDER. Single pink, dark centre.

OLEANDER. Single salmon-yellow.

OLEANDER. Single white.

PITTOSPORUM CRASSIFOLIUM. Tall shrub. Thick leaves, small red flrs. Good for a hedge or windbreak. $2-3 \mathrm{ft}$., pot, $50 \mathrm{c}$ ea., $\$ 4.00$ per 10 ; $3-4 \mathrm{ft}$., pot, $60 \mathrm{c}$ ea.

P. EUGENOIDES. Very bright, light green foliage; makes a beautiful hedge. 10-12 in., pot, $30 \mathrm{c}$ ea., $\$ 2.50$ per $10 ; 2-3$ ft., pot, $50 \mathrm{c}$ ea., $\$ 4.00$ per $10 ; 10-15$ in., can, $20 \mathrm{c}$ ea., $\$ 1.50$ per 10.

P. NIGRICANS. Small, roundish leaves shining. Very good for hedges. 1-2 ft., pot, $20 \mathrm{c}$ ea., $\$ 1.50$ per $10 ; 2-3 \mathrm{ft}$., pot, $50 \mathrm{c}$ ea., $\$ 4.00$ per 10 ; $10-15$ in., can, 20 c ea., $\$ 1.50$ per 10 .

P. TOBIRA. Low growing. Fragrant white flowers. 8-12 in., pot, $35 \mathrm{c}$ ea., $\$ 3.00$ per 10 .

P. TOBIRA VARIEGATA. Leaves silvery. 8-12 in., pot, $35 \mathrm{c}$ ea., $\$ 3.00$ per 10 .

P. UNDULATUM. Leaves large, wavy. Flowers numerous, white, fragrant. Very beautiful. Best near the coast, or in rather sheltered situation. 1-2 ft., pot, $35 \mathrm{c}$ ea., $\$ 3.00$ per 10 , $\$ 25.00$ per $100 ; 2-3$ ft., pot, $50 \mathrm{c}$ ea., $\$ 4.00$ per 10 .

PLUMBAGo. Sky-blue flrs. 1-2 ft., pot, $40 \mathrm{c}$ ea., $\$ 3.50$ per 10 .

PLUMBAGO. White flowers. 1-2 ft., pot, $40 \mathrm{c}$ ea., $\$ 3.50$ per 10 .

PLUMBAGO. Dwarf. Flowers deep blue. $1 \mathrm{ft}$., pot, $35 \mathrm{c}$ ea., $\$ 3.00$ per 10

PACHYSANDRA TERMINALIS. Almost procumbent small shrub. Leaves bright green; flrs. white. Good for rockeries. Pot, $30 \mathrm{c}$ ea., $\$ 2.50$ per 10.

POLYGALA. Small shrub, covered all summer with rich magenta or deep rose colored flrs. Hardy. 1-2 ft., pot, $35 \mathrm{c}$ ea., $\$ 3.00$ per $10 ; 2-3$ ft., pot, $50 \mathrm{c}$ ea., $\$ 4.00$ per 10 .

POMEGRANATE. Dwarf evergreen. Flowers scarlet. New. Rare. 6-12 in., pot, $\$ 1.00$ ea

PRIVET (Ligustrum japonicum). Leaves large, shiny; flrs; white. Very handsome shrub. Hardy. 2-3 ft., pot, $50 \mathrm{c}$ ea., $\$ 4.00$ per $10 ; 3-4$ ft., pot, $60 \mathrm{c}$ ea., $\$ 5.00$ per 10 ; specimen, $3-4$ ft., tub, $\$ 1.50-\$ 2.50$ ea.

PRIVET (L. ovalifolium). California Privet. Best for hedges. 2-3 ft., pot, $30 \mathrm{c}$ ea., $\$ 2.50$ per 10 ; 3-4 ft., pot, 40c ea.; 1-2 ft., open ground, 40c ea., $\$ 3.50$ per 10 ; bushy, $1-2 \mathrm{ft}$., flats, $\$ 7.50$ per 100 .

PRIVET L CILIATUM. Thick leaves, rich green. Handsome shrub. New. 1-2 ft., pot, $50 \mathrm{c}$ ea.

RAPHIOLEPIS OVATA. Compact growing; leaves large, very dark green; flrs. white; berries black. 6-10 in., pot, $40 \mathrm{c}$ ea., $\$ 3.50$ per 10. 
ROCK ROSE CISTUS MACULATA (ladaniferus). Flowers very large, white, with dark crimson dots at base of petals. Likes sunshine. 2-3 ft., pot, $50 \mathrm{c}$ ea., $\$ 4.00$ per 10 .

ROCK ROSE. Crimson flrs. $2-3 \mathrm{ft}$., pot, $40 \mathrm{c}$ ea., $\$ 3.00$ per 10 .

SOLANUM XANTI (Calif). Low shrub, with large violet-blue flrs. $1-2 \mathrm{ft}$., pot, $50 \mathrm{c}$ ea., $\$ 4.00$ per 10.

SOLANUM (Burbank). Similar to above. $1 \mathrm{ft}$., pot, $50 \mathrm{c}$ ea., $\$ 4.00$ per 10 .

SOLANUM PSEUDO-CAPSICUM. Jerusalem Cherry. 6-10 in., pot, $25 \mathrm{c}$ ea.; $1-2 \mathrm{ft}$., pot, $35 \mathrm{c}$ ea., $\$ 3.00$ per 10 .

SUMACH (Rhus ovata). Leaves large; spikes of yellow flrs., red berries. 1-2 ft., pot, $60 \mathrm{c}$ ea., $\$ 5.00$ per $10 ; 3-4 \mathrm{ft}$., 5 gal. can, $\$ 1.00$ ea

SWAINSONIA. Crimson flrs. resembling sweetpea. 1-2 ft., pot, $40 \mathrm{c}$ ea., $\$ 3.50$ per 10 .

SWAINSONIA. White firs. 1-2 ft., pot, $40 \mathrm{c}$

STRAWBERRY TREE (Arbutus unedo). 1-2 ft., pot, $75 \mathrm{c}$ ea., $\$ 6.00$ per $10 ; 2-3 \mathrm{ft}$., 5 gal. can, $\$ 1.25$ ea.

SAGE (Audibertia) Calif. White. 1-2 ft., pot, $40 \mathrm{c}$ ea.

SAGE. Black. 1-2 ft., pot, $40 \mathrm{c}$ ea.

TEA TREE (Leptospermum laevigatum). Leaves small, bluish green. Grows to large shrub, with drooping habit. Flrs. white. Very useful as a screen or as specimen plants. Hardy. 3-4 ft., 1 gal. can, $60 \mathrm{c}$ ea., $\$ 5.00$ per $10 ; 4-6 \mathrm{ft}$., 1 gal. can, $75 \mathrm{c}$ ea., $\$ 6.00$ per 10 .

TEA TREE (L. lanigerum). Leaves narrow; firs. larger. 3-4 ft., pot, 60c ea.

THORN (Crataegus crenulata). Burning Bush. Large shrub or tree. Flowers white, followed by profusion of scarlet berries. Hardy. Very useful anywhere. 3-4 ft., pot, $60 \mathrm{c}$ ea., $\$ 5.00$ per 10 ; flats, $\$ 7.50$ per 100 .

THORN (C. pyracantha). Burning Bush. Berries large, orange-red. 1-2 ft., pot, $40 \mathrm{c}$ ea., $\$ 3.50$ per 10 .

THORN (C. pyracantha-lalandi). Of slender habit. 2-3 ft., pot, $60 \mathrm{c}$ ea., $\$ 5.00$ per 10 .

THORN (C. yunnanensis). Bright, glossy leaves; berries coral red. New. 2-3 ft., pot, 60c ea.

TREE ANEMONE (Carpenteria Californica). Handsome native shrub, with very large fragrant white flowers, and prominent yellow stamens. 4-6 in., pot, $35 \mathrm{c}$ ea., $\$ 3.00$ per 10 ; 1-2 ft., pot, $75 \mathrm{c}$ ea.
THUJA BOREALIS GLAUCA. Erect, compact habit. Foliage blue-green; very distinct. Useful for formal planting or porch decoration. 1-2 ft., balled, $\$ 1.00$ each; 3 ft., 5 gal. can, $\$ 2.50$ ea.; $31 / 2$ ft., 5 gal. can, $\$ 3.00$ ea.

THUJA COMPACTA. Similar habit to above, but with light, golden green foliage, and taller growing. 4-5 ft., 5 gal. can, $\$ 3.00$ ea.; 5-6 ft., 5 gal. can, $\$ 3.50$ ea.; 7 ft., 5 gal. can, $\$ 4.00$ ea.

VERONICA ANDERSONI. Strong grower flowers purple. Hardy. 2-3 ft., 1 gal. can or pot, $60 \mathrm{c}$ ea.. $\$ 5.00$ per 10 .

VERONICA ÄLBA. Flowers white; upright grower. $1-2 \frac{1 / 2}{\mathrm{ft}}$, pot, $50 \mathrm{c}$ ea., $\$ 4.00$ per 10 . $2-3 \mathrm{ft}$., pot, $60 \mathrm{c}$ ea., $\$ 5.00$ per 10 .

VERONICA CUPRESSOIDES. Leaves needlelike; flowers lilac; distinct. New. 6-8 in., pot, $50 \mathrm{c}$ ea.

VERONICA DECUSSATA. Foliage light; flowers white to pink. $1 \mathrm{ft}$., pot, $40 \mathrm{c}$ ea.; $3-4 \mathrm{ft} ., 5$ gal. can, $\$ 1.50$ ea.

VERONICA IMPERIALIS. Flowers purplishblue; free flowering; one of the best. 1-2 ft., pot, $50 \mathrm{c}$ ea., $\$ 4.00$ per $10 ; 2-3 \mathrm{ft} .$, pot, $60 \mathrm{c}$ ea., $\$ 5.00$ per 10 .

VERONICA TRAVERSII. Dense; low growing; flowers white. 1-2 ft., pot, $40 \mathrm{c}$ ea.

VERONICA VARIEGATA. Foliage creamy white; flowers lilac. The Veronicas are unexcelled for grouping. Succeed anywhere, if not allowed to get too dry in the summer. 1-2 ft., pot, $50 \mathrm{c}$ ea., $\$ 4.00$ per 10 .

VIBURNUM JAPONICUM. Leaves very large; rich green; flowers white. Grows to large shrub. Fine for a lawn. 10-15 in., pot, $40 \mathrm{c}$ ea., $\$ 3.50$ per 10 . Specimen plants. $4-6 \mathrm{ft}$. 5 gal. can., $\$ 3.50$ ea.

WILD BROOM (Hosackia). California. Grows well in dry places; very graceful. 1-2 ft., pot, $50 \mathrm{c}$ ea., $\$ 4.00$ per 10 .

WILD BUCKWHEAT (Erigeron). California. Very pretty native shrub, covered all summer with racemes of pinkish flowers. 1-2 ft., pot, $50 \mathrm{c}$ ea.

WILD ORANGE (Prunus Caroliniana). Allied to our Evergreen Cherry. Leaves large; flowers white, in dense clusters; fruit black. $1-2 \mathrm{ft}$., pot, $40 \mathrm{c}$ ea., $\$ 3.50$ per $10 ; 2-3 \mathrm{ft}$., pot, $60 \mathrm{c}$ ea., $\$ 5.00$ per 10 .

\section{CLIMBERS}

AKEBIA QUINATA. Leaves bright; flowers rosypink; fragrant. Hardy. Evergreen. pot, $40 \mathrm{c}$ ea., $\$ 3.50$ per 10 .

AMPELOPSIS INCONSTANS LOWII. Leaves finely cut, deep green, turning red. New. Deciduous. Pot, $40 \mathrm{c}$ ea., $\$ 3.50$ per 10 .

AMPELOPSIS OUINOUIFOLIA. $\mathrm{V}$ i $\mathrm{r} \mathrm{g}$ i $\mathrm{n}$ i a Creeper. Deciduous. Pot, $35 \mathrm{c}$ ea., $\$ 3.00$ per 10 .

AMPELOPSIS SEMPERVIRENS. Evergreen Boston Ivy. Makes fine ground cover. Pot, $35 \mathrm{c}$ ea., $\$ 3.00$ per 10

AMPELOPSIS VEITCHII. Boston Ivy. Leaves turn red. Self-clinging. Pot, $35 \mathrm{c}$ ea., $\$ 3.00$ per 10.

ASPARAGUS PLUMOSUS. For hanging baskets or outdoors along coast. Pot, $35 \mathrm{c}$ ea., $\$ 3.00$ per 10. Large specimens. Pot, $75 \mathrm{c}-\$ 1.25$ ea.

ASPARAGUS SPRENGERI. For hanging baskets, or cover for trellis near coast. Pot, $35 \mathrm{c}$ ea., $\$ 3.00$ per 10 . Large specimens. Pot, $75 \mathrm{c}-\$ 1.25$ ea.

BIGNONIA CHERERE. Flowers red, trumpet shaped. Evergreen. Fairly hardy. Pot, $40 \mathrm{c}$ ea.

BIGNONIA GRANDIFLORA. Flowers large, red, trumpet shaped. Deciduous. Pot, $40 \mathrm{c}$ ea., $\$ 3.50$ per 10 .
BIGNONIA RADICANS. Similar, but flowers smaller. Pot, 35c ea., $\$ 3.00$ per 10 .

BIGNONIA TWEEDIANA. Flowers yellow, Rather tender. Evergreen. Pot, $40 \mathrm{c}$ ea., $\$ 3.50$ per 10

BIGNONIA VIOLACEA. Flowers large, lilac, Evergreen. Hardy. Pot, $40 \mathrm{c}$ ea.

BOUGAINVILLEA. Flowers crimson; very showy. Rather tender. Evergreen. Pot, $60 \mathrm{c}$ ea.

BOUSSINGAULTIA. Madeira vine. Strong grower; tuberous rooted; perennial. Flowers white. Pot, $40 \mathrm{c}$ eq.

BRIDGESIA SPICATA. Beautiful vine; flowers rosy-pink; leaves rich green; clings to tree. Hardy. Pot, $40 \mathrm{c}$ ea., $\$ 3.50$ per 10 .

CLEMATIS CALIFORNICA. Virgin's Bower. D. Pot, $35 \mathrm{c}$ ea., $\$ 3.00$ per 10 .

CLEMATIS JACKMANNI. Flowers deep purple, large. D. Pot, $60 \mathrm{c}$ ea.

CLEMATIS MONTANA. Vigorous; flowers large, white, in clusters, fragrant. Pot, $40 \mathrm{c}$ ea., $\$ 3.50$ per 10 .

CLEMATIS LILACINA. Variety of above, lilac flowers. Pot, $50 \mathrm{c}$ ea.

CLEMATIS RUBENS. Flowers reddish. Pot, $50 \mathrm{c}$ ea. 
CLEMATIS PANICULATA. Japanese... Flowers white, fragrant. Ev. Pot or cans, $40 \mathrm{c}$ ea., $\$ 3.50$ per 10 .

CLIANTHUS. Parrot's Bill. Flowers white, or red. Pot, $35 \mathrm{c}$ ea.

DOLICHOS. Australian Pea vine. Flowers deep pink. Vigorous. Pot, $35 \mathrm{c}$ ea., $\$ 3.00$ per 10 .

FICUS REPENS. Climbing Fig. Clings to walls. Ev. Pot, $35 \mathrm{c}$ ea., $\$ 3.00$ per 10 .

GELSEMIUM. Carolina Yellow Jessamine. Pot, $35 \mathrm{c}$ ea.

HEDERA. English Ivy. Splendid assortment. Hardy. Pot, $35 \mathrm{c}$ ea., $\$ 3.00$ per 10 .

HEDERA AMURENSIS. Very large leaves; light green. Pot, $35 \mathrm{c}$ ea.. $\$ 3.00$ per 10 .

HEDERA ANGULARIA-AUREA. Finely cut. Golden Green. Pot, $35 \mathrm{c}$ ea., $\$ 3.00$ per 10 .

HEDERA AZORICA. Leaves large, glaucous green. Pot, $35 \mathrm{c}$ ea., $\$ 3.00$ per 10 .

HEDERA DENTATA. Very large leaves. Pot, $35 \mathrm{c}$ ea., $\$ 3.00$ per 10 .

HEDERA DENTATA AUREA. Very large leaves, broad border of gold. Rare. Pot, $50 \mathrm{c}$ ea.

HEDERA. Emerald Gem. Small leaves; very dark green. Pot, $35 \mathrm{c}$ ea., $\$ 3.00$ per 10 .

HEDERA. Common English. Strong grower. Pot, $35 \mathrm{c}$ ea., $\$ 3.00$ per 10 .

HEDERA. Tree, flowering. Pot, $50 \mathrm{c}$ ea.

HEDERA. Japan tri-color; variegated foliage;

Small. Pot, $50 \mathrm{c}$ ea.
HEDERA MARGINATA ELEGANS. Leaves small, edged with silvery-gold. Pot, $50 \mathrm{c}$ ea.

HEDERA. Mrs. Pollock. Leaves very small, finely cut. Pot, $35 \mathrm{c}$ ea, $\$ 3.00$ per 10 .

HEDERA REGNERIANUM. Dark, roundish leaves. Pot, $35 \mathrm{c}$ ea., $\$ 3.00$ per 10 .

JASMINE BESSONIANUM. Flowers deep rose, fragrant. New. Ev. Hardy. Pot, $50 \mathrm{c}$ ea

JASMINE OFFICINALE. Flowers white, fragrant. Ev. Hardy. Pot, $35 \mathrm{c}$ ea., $\$ 3.00$ ea.

JASMINE PRIMULINIUM. Flowers yellow, large; leaves large. Hardy. Ev. Pot, 35c ea., $\$ 3.00$ per 10.
JASMINE REVOLUTUM. Italian yellow Jessamine. Ev. Pot, $35 \mathrm{c}$ ea.

JASMINE SIMPLICIFOLIUM. Vigorous. Flowers white. Ev. Pot. $40 \mathrm{c}$ ea.

LONICERA CALIFORNICA. Wild Honeysuckle. D. Pot, $35 \mathrm{c}$ ea.

LONICERA CHINENSIS. Strong grower. Leaves reddish; flowers creamy, fragrant. Ev. Pot, $35 \mathrm{c}$ ea., $\$ 3.00$ per 10.

LONICERA JAPONICA. Hall's Honeysuckle. Fragrant. Ev. Pot, 35c ea., \$3.00 per 10.

MANDEVILLA. Very fragrant pure white flowers. Vigorous. Hardy. Deciduous. Pot, $35 \mathrm{c}$ ea., $\$ 3.00$ per 10 .

MUEHLENBECKIA. Wire Vine. Extremely useful as a porch screen. Leaves very small. Hardy. Pot, $35 \mathrm{c}$ ea., $\$ 3.00$ per 10 .

POLYGONUM BALDSCHUANICUM. Strong grower. Large panicles white flowers. Hardy. D. Pot, $40 \mathrm{c}$ ea., $\$ 3.50$ per 10 .

POLYGONUM AUBERTII. Similar. Leaves reddish. New. $50 \mathrm{c}$ ea.

RUBUS ELLIPTICUS. Evergreen Raspberry. Very strong grower; frower; fruit yellow; stems covered with red hairs. Ornamental. Balled. $50 \mathrm{c}$ ea.

SOLLYA HETEROPHYLLA. Blue-bell vine. Hardy. E. Pot, $40 \mathrm{c}$ ea., $\$ 3.50$ per 10 .

PUERARIA HIRSUTA. Kudzu Vine. Immense leaves; enormous grower. Perennial. Pot, $40 \mathrm{c}$ ea.

PASSIFLORA CAERULEA. Passion vine. Flowers bluish; hardy; evergreen. Pot, $35 \mathrm{c}$ ea.

P. INCANATA. Flowers white, purple corona. Ev. Pot, $40 \mathrm{c}$ ea.

SOLANUM JASMINOIDES. Potato vine; strong grower, flowers lilac in cluster; hardy. Ev. Pot, $35 \mathrm{c}$ ea., $\$ 3.00$ per 10 .

VITIS HENRYI. Resembles Virginia Creeper, but with striped leaves; vigorous. D. Pot, $35 \mathrm{c}$ ea., $\$ 3.00$ per 10

WISTERIA. In blue, white and lavender. Pot, $60 \mathrm{c}$ ea.

\section{PERENNIALS AND POT PLANTS}

ACANTHUS MOLLIS. Handsome decorative plant for lawn or border; perennial. Balled or pot, $35 \mathrm{c}$ ea.

ACTAEA ARGUTA. Baneberry. California white flowers, red berries. Pot, $40 \mathrm{c}$ ea.

AGAVE. Century plant. Pot, $35 \mathrm{c}$ ea.

AGAVE. Variegated leaves; pot or tub culture. Pot, $40 \mathrm{c}$ ea:

ALOYSIA. Lemon Verbena. Pot, $35 \mathrm{c}$ ea.

ARABIS. Rock Cress-Rockeries. Pot, 35c ea ARALIA CALIFORNICA. Spikenard. Moist location. Pot, $40 \mathrm{c}$ ea.

A. SIEBOLDII. Very large, shiny leaves. Pot culture. 5 gal. can, $\$ 1.25$.

ARMERIA. Sea Pink. Border plant. Balled, $25 \mathrm{c}$ ea., $\$ 1.50$ per $10, \$ 10.00$ per 100

ARTEMISIA. Dusty Miller. Pot, $25 \mathrm{c}$ ea., $\$ 2.00$ per 10.

ASARUM. Wild Ginger. Round leaves, white flowers; shady spot, moist. Pot, $35 \mathrm{c}$ ea.

ASPARAGUS ELONGATUM. Upright, feathery very useful for bouquets or pot culture. New. Pot, $50 \mathrm{c}$ ea.

ASPIDISTRA. Very large leaves. Best plant for indoors. Pot, $50 \mathrm{c}-75 \mathrm{c}$ ea, tub $\$ 1.25$.

ASTER. Michaelmas Daisy. Perennial. Pot, $35 \mathrm{c}$ ea.

BEGONIA. Bedding varieties; pink and rose.

Pot, $35 \mathrm{c}$ ea.

CALADIUM. Elephant's Ear.
conservatory. Pot, 50c ea.

CAMPANULA. Blue-bells. Pot, $35 \mathrm{c}$ ea.

CHAENOSTOMA. Border plant. Flowers small, lilac. Ev. Pot, $25 \mathrm{c}$ ea., $\$ 2.00$ per 10.

CORDYLINE. Dracena Palm. 5 gal. can $\$ 1.50$ $\$ 12.50$ per 10 , tub $\$ 3.50$.
CORDYLINE TERMINALIS. Foliage plant for porch. Pot, 50c ea. C. MARITIMA. California Sea Dahlia. Pot, $35 \mathrm{c}$ ea.

CYNARA. Artichoke. Used as a vegetable. Pot, $25 \mathrm{c}$ ea., $\$ 2.00$ per 10 .

ERIOPHYLLUM. California Yarrow. Yellow flowers. Pot, $35 \mathrm{c}$ ea.

ERYSIMUM. California Coast Wall-flower. Flowers creamy. Pot, $35 \mathrm{c}$ ea.

EULALIA GRACILLIMUS. Ornamental grass. Can or clump, $50 \mathrm{c}$ ea.

E. ZEBRINUS. Ornamental grass. Leaves striped. Can or clump, 50c ea.
FELICIA. Blue Marguerite. Pot, 30 c ea., $\$ 2.50$ FELICIA.

FERNS. Native ferns in variety; $35 \mathrm{c}$ ea., $\$ 3.00$ per 10.

FUCHSIA. Assorted varieties. Pot, $35 \mathrm{c}$ ea.,

$\$ 3.00$ per 10.
GAILLARDIA. Flowers large, red and yellow. Pot, $30 \mathrm{c}$ ea., $\$ 2.50$ per 10 .

GYPSOPHYLLA. Baby breath. Pot, $35 \mathrm{c}$ ea., $\$ 3.00$ per 10 .

HELIOTROPE. Lavender, purple, white. Pot, $30 \mathrm{c}$ ea.

HEUCHERA (California). Leaves roundish, flowers white, on slender stems. Pot, $35 \mathrm{c}$ ea., $\$ 3.00$ per 10.

H. SANGUINEA. Same, with red flowers. Pot, $50 \mathrm{c}$ ea.

HUNNEMANNIA. Mexican Poppy. Flowers large, primrose yellow. Pot, $30 \mathrm{c}$ ea., $\$ 2.50$ per 10. 
KNIPHOFIA. Red-hot Poker. Pot, 35 c ea., $\$ 3.00$ per 10.

LANTANA. Pink, orange, yellow, white. Pot, 35 c ea., $\$ 3.00$ per 10 .

LAVANDULA. Lavender, lilac or white. Pot or can, $35 \mathrm{c}$ ea., $\$ 3.00$ per 10 .

LUPINUS ARBOREUS. Yellow Tree Lupine. Pot, $40 \mathrm{c}$ ea.

MENTHA. Mint. Pot, $30 \mathrm{c}$ ea.

MESEMBRYANTHEMUM ARBOREUS . Grows into large, woody shrub; flowers yellow. Pot, $30 \mathrm{c}$ ea., $\$ 2.50$ per 10 .

PAPAVER NUDICAULE. Iceland Poppy. Pot, $25 \mathrm{c}$ ea., $\$ 2.00$ per 10 .

P. ORIENTALE. Oriental Poppy. Pot, 25c ea., $\$ 2.00$ per 10 .

PELARGONIUM. Geranium. In variety. Pot, $30 \mathrm{c}$ ea.

PENSTEMON ANTIRHINOIDES. California. Yellow. Pot, $40 \mathrm{c}$ ea., $\$ 3.50$ per 10 .

P. CENTRANTHIFOLIUS. California. Scarlet. Pot, $40 \mathrm{c}$ ea., $\$ 3.50$ per 10 .

P. HETEROPHYLLUS. California. Purplish. $40 \mathrm{c}$ ea., $\$ 3.50$ per 10 .

P. HYBRIDUM. Flowers large, white or crimson. Pot, $40 \mathrm{c}$ ea.

PHORMIUM TENAX New Zealand Flax. Long, sword-like leaves. Pot or tub culture. Pot, $50 \mathrm{c}$ ea.; tub, $\$ 1.25$.
ROSEMARY. $30 \mathrm{c}$ ea.

RUDBECKIA. Golden Glow. Can, $30 \mathrm{c}$ ea.

SEA ASTER. Stokesia. Flowers large, lilac. Pot, $35 \mathrm{c}$ ea.

SEA MARIGOLD. Flowers large, yellow; border plant. In fiats, $10 \mathrm{c}$ ea., $75 \mathrm{c}$ per 10.

SEDUM. Stone crop, in variety. In flats, 50c per 10.

SHASTA DAISY. Can or pot, $25 \mathrm{c}$ ea.

SISYRINCHIUM CALIFORNIA. Blue-eyed grass. Pot, $30 \mathrm{c}$ ea.

THYME. Aromatic. Pot or can, $25 \mathrm{c}$ ea.

VIOLET. Belle de Chatenay White, rosy edge. $10 \mathrm{c}$ ea, $75 \mathrm{c}$ per 10 .

VIOLET. Czar. Single white. $10 \mathrm{c}$ ea., $75 \mathrm{c}$ per 10.

VIOLET. J. Roddenberg. Light blue. $10 \mathrm{c}$ ea., $75 \mathrm{c}$ per 10

VIOLET. Mrs. Astor. Violet, tinged red. $10 \mathrm{c}$ ea., $75 \mathrm{c}$ per 10 .

VIOLET. Princess of Wales. Deep violet. $10 \mathrm{c}$ ea., $75 \mathrm{c}$ per 10 .

VIOLET. Reine Augustine. Dark violet. $10 \mathrm{c}$ ea., $75 \mathrm{c}$ per 10 .

VIOLET. Swanley White. 10c ea., $75 \mathrm{c}$ per 10.

\section{TRAILING OR CREEPING PLANTS}

\section{CONVOLVULUS MAURITANICA. Blue. Pot,} $40 \mathrm{c}$ ea., $\$ 3.50$ per 10 .

FRAGARIA. Trailing Strawberry. $10 \mathrm{c}$ ea., $75 \mathrm{c}$ per $10, \$ 5.00$ per 100 .

HELIANTHEMOS or SUN-ROSE. Flowers white, pink, yellow, rose, or copper. Very desirable; 25 c ea., $\$ 2.00$ per $10 ; \$ 15.00$ per 100 .

LIPPIA REPENS. ( Flat makes several hundred plants). Flat, $\$ 1.50$.

MESEMBRYANTHEMUM. In variety. $10 \mathrm{c}$ ea., 75 c per $10, \$ 5.00$ per 100 .

M. AURANTICUM. Flowers deep coppery crimson. $20 \mathrm{c}$ ea., $\$ 1.50$ per $10, \$ 10.00$ per 100 .

MICROMERIA (Yerba buena). Aromatic. Pot, $35 \mathrm{c}, \$ 3.00$ per 10
TRADESCANTIA. Wandering Jew. $10 \mathrm{c}$ ea., $75 \mathrm{c}$ per 10 .

VERBENA ERINOIDES. White or purple. Pot, $25 \mathrm{c}, \$ 2.00$ per 10 .

VERBENA. Garden verbena. Assorted colors. Pot, $15 \mathrm{c}, \$ 1.00$ per 10 .

VERBENA VENOSA. Taller growing. Pot, 25 c ea.

VINCA MAJOR VARIEGATA. Foliage green and gold; flowers large, blue. $\$ 5.00$ per 100 .

VINCA MINOR. Blue; smaller, leaves dark green. $\$ 7.50$ per 100 .

VINCA MINOR. White. $\$ 7.50$ per 100 .

VINCA MINOR . Variegated. Leaves golden. $\$ 7.50$ per 100 .

\section{CANNAS}

We call attention to our splendid collection of Orchid flowering and decorative Cannas. Price in pots, $30 \mathrm{c}$ ea., root clumps, $20 \mathrm{c}$ ea., $\$ 1.50$ per 10 .

CANNA ALBA ROSEA. A most unusual and lovely canna; flowers small, with stiff narrow petals, the loveliest peach blossom shading to creamy white. 3-4 $\mathrm{ft}$.

ALLEMANNIA. Orange color, very large, spotted with red. 5-6 $\mathrm{ft}$.

AUSTRIA. Yellow, very large. 3-4 ft.

CRIMSON BEDDER. Rich scarlet; large; 3-4 ft.

DAVID HARUM. Red, foliage bronze-tinted. $3 \mathrm{ft}$.

F. BENARY. Fiery orange, yellow edge. 5-6 ft.

GLADIATOR. Yellow, spotted and splashed with erimson.

KARL MERCK. Large, pale yellow, streaked pink at throat. $3-4 \mathrm{ft}$.
KING HUMBERT. Large, scarlet often streaked with gold; foliage bronze. $31 / 2 \mathrm{ft}$.

KING OF BRONZES. Small, red; foliage rich bronze. $6-7 \mathrm{ft}$.

LOUISE. Large, pink, tinges salmon. 3-4 ft.

LOUISIANA. Deep red; 4-5 ft.

MEPHISTO. Dark red, 3-4 $\mathrm{ft}$.

MOONLIGHT. Small creamy-white, 3-4 ft.

MUSAFOLIA. Small leaves; foliage dark green, edged bronze; 7-8 $\mathrm{ft}$.

PENNSYLVANIA. Deep crimson; very large, 5-6 ft. PRINCE WEID. Scarlet; yellowish tinge. 5-6 ft. VENUS. Pink and gold; very large. 3-4 $\mathrm{ft}$.

WM. SAUNDERS. Red, rather small; foliage. $3 \frac{1}{2} \mathrm{ft}$. 


\section{ROSES}

Roses thrive best in a rather heavy, moist soil, and require good and frequent watering in the summer. After the first year, plenty of manure should be used on or near the surface, not about the roots. Prune heavily every year, keeping the old wood cut out, and shortening what is left. Our Roses are on their own roots in most cases. No trouble from wild root suckers. The following list comprises the best in all colors and habits for California. Prices are for strictly No. 1 stock:

AMERICAN BEAUTY. H. P. Rosy flesh on yellow ground. $35 \mathrm{c}$ each.

BABY LYON. P. Coral, shaded yellow. $75 \mathrm{c}$ ea. BABY PHYLLIS. P. Carmine pink in large panicles. Novel and distinct. $35 \mathrm{c}$ ea.

BETTY. H. T. Coppery. Fragrant. 35c ea.

BRITISH QUEEN. Pure white. $35 \mathrm{c}$ ea.

CHEROKEE. Pink. $35 \mathrm{c}$ ea.

CHEROKEE. White. $35 \mathrm{c}$ ea.

CLIMBING CECILE BRUNNER. Small flowers, clear pink. $35 \mathrm{c}$ ea.

CLIMBING HOOSIER BEAUTY. H. T. Dark crimson; best. $40 \mathrm{c}$ each.

CLIMBING KAISERIN. White. $40 \mathrm{c}$ ea.

CLIMBING GENERAL MacARTHUR. Deep red. $\$ 1.00$ ea.

CLIMBING LIBERTY. Velvety crimson. $35 \mathrm{c}$. ea. CLIMBING SUNBURST. H. T. Deep yellow, orange center. $40 \mathrm{c}$ ea.

ETOILE DE FRANCE. H. T. Ruby red. $35 \mathrm{c}$ ea.

FRAU K. DRUSCHKI. H. P. Snow-white. $35 \mathrm{c}$ ea.

GEN. JACQUEMINOT. H. P. Crimson-scarlet; very fragrant. $35 \mathrm{c}$ ea.

GEN. MacARTHUR. H. T. Bright scarlet. $35 \mathrm{c}$ ea.

GEO. ELGER. P. A. yellow "Cecile Brunner." $40 \mathrm{c}$ e 2 .

GOLD OF OPHIR. Combination of copper, carmine and salmon. $35 \mathrm{c}$ ea.

HOOSIER BEAUTY. H. T. Deep crimson, with darker shading. $40 \mathrm{c}$ ea.

IRISH ELEGANCE. H. T. Single, bronzy-red, shading to apricot. $35 \mathrm{c}$ ea.
IRISH FIREFLAME. H. T. Old gold, shaded crimson. $35 \mathrm{c}$ ea.

LA FRANCE. Sat'n pink. $35 \mathrm{c}$ ea.

LADY HILLINGDON. Deep orange-yellow. $50 \mathrm{c}$ ea.

I.AMARQUE. Climber. Double white. $35 \mathrm{c}$ ea.

I.AMARQUE. Climber. Double white. $35 \mathrm{c}$ ea.
MAMAN COCHET. T. salmon-yellow. $\quad 25 \mathrm{c}$ ea.

MME. A. CHATENAY. H. T. Carmine-rose.

$35 \mathrm{c}$ ea.
MME. C. TFSTOUT. H. T. Clear pink. $35 \mathrm{c}$ ea.

MME. C. BRUNNER. P. Bright rose, shaded salmon-pink. $35 \mathrm{c}$ ea.

MARECHAL NIEL. Deep, golden yellow; climber; fragrant. $75 \mathrm{c}$ ea.

MME. ED. HERRIOTT. H. T. Terra-cotta or reddish-yellow. $50 \mathrm{c}$ ea.

MRS. AARON WARD. H. T. yellow center. Pink edge. $35 \mathrm{c}$ ea.

MRS. CHAS. BELL. H. T. Shell-pink. Very distinct. $75 \mathrm{e}$ ea.

LOS ANGELES. H. T. Flame-pink, toned with coral, shaded gold at base of petals. $\$ 1.00$ ea.

OPHELIA. H. T. Salmon-rose. $35 \mathrm{c}$ ea.

OTHELLO. H. P. Deep crimson. $40 \mathrm{c}$ ea.

PAPA GONTIER. T. Rosy-crimson. $35 \mathrm{c}$ ea.

PERLE D'OR. P. Nankeen yellow. $35 \mathrm{c}$ ea.'

RADIANCE RED. Enormous globular flowers, dazzling crimson scarlet. $50 \mathrm{c}$ ea.

RADIANCE. H. T. Rosy opaline-pink. $35 \mathrm{c}$ ea.

RAINBOW. Deep Pink, splashed with carmine. $35 \mathrm{c}$ ea.

SAFRANO. T. Bright apricot. $35 \mathrm{c}$ ea.

SHOWER OF GOLD. Deep golden yellow; climber; very double. $35 \mathrm{c}$ ea.

SUNBURST. H. T. Deep yellow; orange center. $50 \mathrm{c}$ ea.

WHITE CECILE BRUNNER. White, tinted flesh. $40 \mathrm{c}$ ea.

WHITE BANKSIA. Small white flowers. $40 \mathrm{c}$ ea. WHITE C. TESTOUT. Pure white. $40 \mathrm{c}$ ea.

YELLOW BANKSIA. $40 \mathrm{c}$ ea.

\section{PALMS}

CHAMEROPS EXCELSA. Windmill Palm. Very hardy, and one of the best for porch decoration or for outdoors. In tubs, 1-2 ft., $\$ 1.50$ ea. 2-3 $\mathrm{ft}$. $\$ 2.00$ ea. In $1-\mathrm{gal}$. cans, $1-2 \mathrm{ft}$. $\$ 1.00$ ea. Quart cans, $8-12$ in. $60 \mathrm{c}$ ea.; $\$ 5.00$ per 10 .

CHAMEROPS HUMILIS. Dwarf growing; quite hardy; for porch decoration. Fine specimens in tubs, $2-3 \mathrm{ft}$. $\$ 3.00$ ea. $3 \mathrm{ft}$., $\$ 3.50$ ea.

Cocos AUSTRALIS. Pindo Palm. Very graceful and suitable for porch or outdoor culture. Quite hardy. 2-3 ft., in box or tub, $\$ 3.00$ ea. $3 \mathrm{ft}$., $\$ 3.50$ ea.
ERYTHEA ARMATA. Blue Palm. Very handsome; lvs., fan shaped. Quite hardy. 2-3 ft. in box, 5-gal. can or tub, $\$ 3.00$ ea.

ERYTHEA EDULIS. Guadalupe Island Palm Very hardy; lvs. fan shaped, handsome. 2-3 $\mathrm{ft} ., \$ 2.50$ ea. $3 \mathrm{ft} ., \$ 3.00$ ea. $3-4 \mathrm{ft}$., $\$ 3.50$

ea. 5 ft., $\$ 5.00$ ea.
INBCEA SPECTABILIS. Honey Palm. Very handsome specimens. 2-3 ft., in boxes, $\$ 3.50$ ea. Quite hardy.

PHOENIX CANARIENSIS. Canary Island Palm. Large, spready; quite hardy. $2-3 \mathrm{ft}$., $\$ 2.50$ ea. $3-4 \mathrm{ft}$., $\$ 3.00$ ea.

\section{BAMBOO}

METAKE. Best tall growing for ornamental pur-

FALCATA. Leaves finer. Both hardy. Large clumps, $75 \mathrm{c}$ ea. In 5 -gal cans, $\$ 1.50$ ea.
DWARF. Clumps, $\$ 1.00$ ea.

ARUNDO DONAX or GIANT REED. Excellent for screen or windbreak. Large clumps, $75 \mathrm{c}$ ea. 


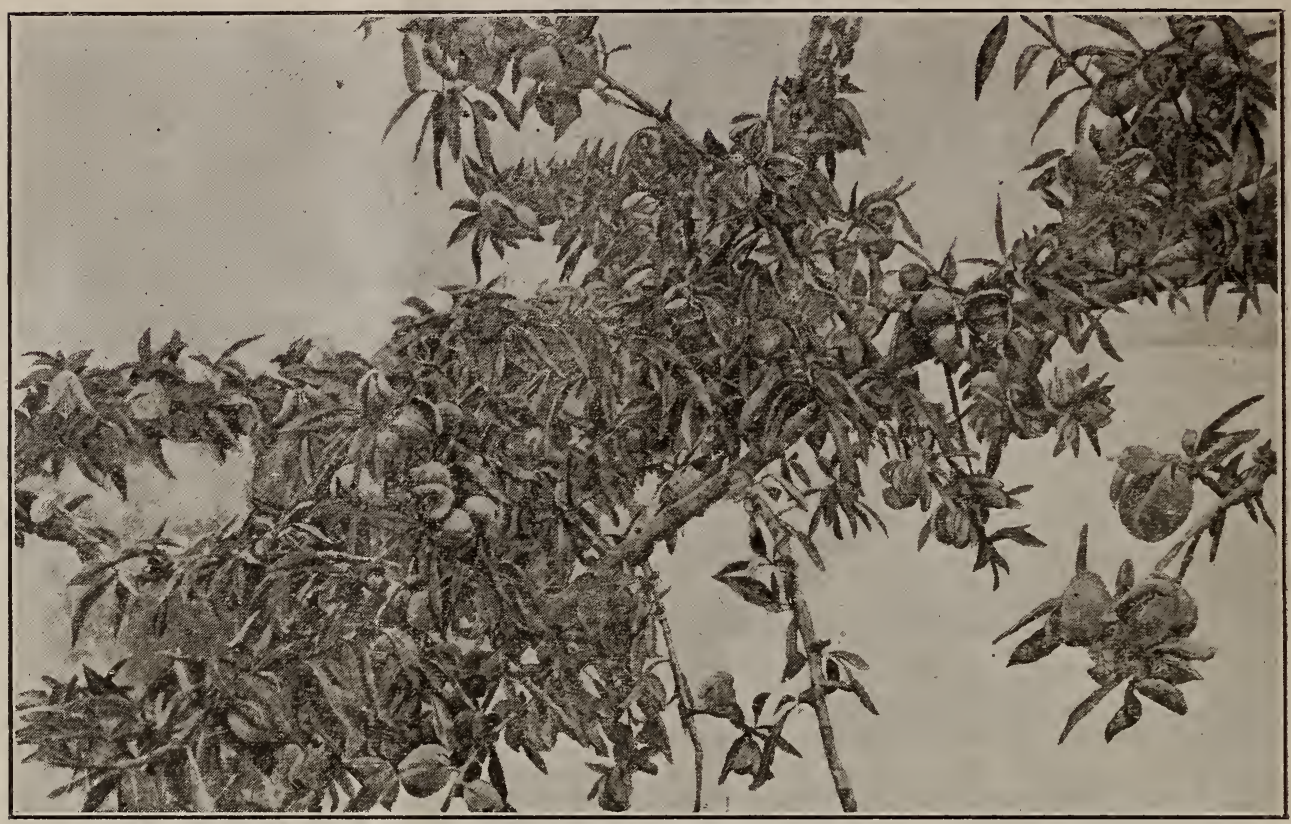

IMPROVED I-XL ALMOND

As helpful to those of our customers who are not familiar with the names and habits of certain shrubs, trees and vines to produce a given effect, we submit the following lists, with some suggestions. We purposely omit many really tender plants which, while they may grow well in Southern California for years, will in time be hurt by frost.

\section{EVERGREEN SHRUBS}

(a) Berried effects; tall.

Some of these are not truly evergreen, but become practically so in California.

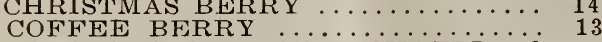
COTONEASTER, FRANCHETTI \& PANNOSA DWARF JAPAN CHERRY ....... 12

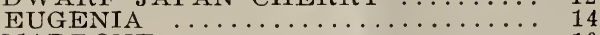

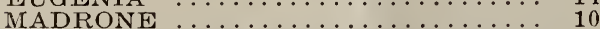

STRAWBERRY TREE ......... 16

THORN, C. CRENULATA and PYRA. CANTHA ................ 16

(b) Berried effects: medium.

BARBERRY, B. DARWINI, ELEGAN'TISSIMA. WILSONEA. STENOPHYLLA and AQUIFOLIUM ...... COTONEASTER ANGUSTIFOLIA $\ldots$. MYRTLE, M. COMMUNIS ......... 15 NANDINA $\ldots \ldots \ldots \ldots \ldots \ldots \ldots \ldots$

Low or Procumbent Habit

COTONEASTER HORIZONTALIS and

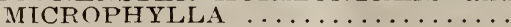
MANZANITA, A. UV̈A-URं

\section{For Ground Covers}

The preceding and AMPELOPSIS SEMPERVIRENS ..... 16 ENGLISH IVIES $\ldots \ldots \ldots \ldots \ldots \ldots \ldots . \ldots 17$ EUONYMUS RADICANS and SIEBOLDI .................... 14

FRAGARIA .............. 18

HYPERICUM, yellow flowers ........ 15

JASMINE PRIMULINIUM, yellow flirs 17

LANTANA (cut down by frost), vari-

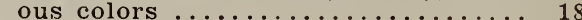

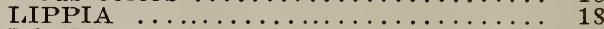

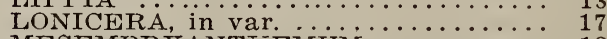

MESEMBRYANTHEMUM ......... is

MICROMERIA ................ 18

PLUMBAGO, blue or white flrs...... 15 ROCK ROSE, various colors ......... 15 SOLLYA, blue flrs. ............ 17

SUN ROSE, various colors …...... 18

VINCA, blue or white ............. 18

\section{Hedges, Borders-Low Growing}

BARBERRY, Evergreen and decidu-

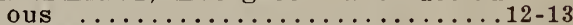
BOX, for low formal borders ....... CATALINA ISLAND CHERRY ....... CYPRESS, for tall hedges ......... 9 EUONYMUS, strong hedge, sunshine.. 14 HEA'TH, low border, cool climate .... 14 
LONICERA, Hall's or Native ........ Page

MT. LILAC, CEANOTHUS SPINOSUS', blue flrs. very good strong hedge... MYRTLE PITOSPORUM, $\cdots \ldots \ldots \ldots \ldots \ldots \ldots$ severai PITTOSPORUM, several varieties,
rather tall $\ldots \ldots \ldots \ldots \ldots \ldots \ldots$ ROCK ROSE, various colors .........

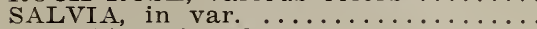
THORN, white flrs.

VERONICA, in var. $\ldots \ldots \ldots \ldots \ldots \ldots$

WILD CHERRY $\ldots \ldots \ldots \ldots \ldots \ldots \ldots$

\section{Cone Bearing Trees}

"BIG TREE"

CEDAR DEODARA. Large, bluish foliCEDAR ÄTLANTiC̈. Tali, symmet-

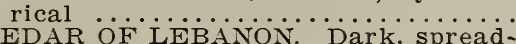
CEDAR OF LEBANON. Dark, spread CYPRESS, ARIZONA. Bluish foliage. CYPRESS, MONTEREY. Rich green. JAPAN REDWOOD $\ldots \ldots \ldots \ldots \ldots \ldots$ NUTMEG TREE. Dark green .......... PINE, MONTEREY. Fast grower ...

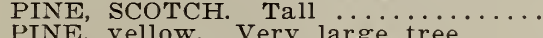
PINE, yellow. Very large tree.....

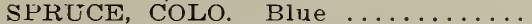
SPRUCE, NORWAY. For Christmas

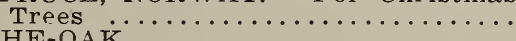

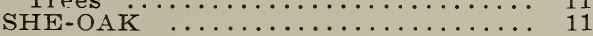

Nutmeg and She-Oak, similar in general appearance, may be classed with the Conifers.

\section{Deciduous Shrubs-flowering}

All of these should be pruned well after flowering.

BARRERRY, B. THUNBERGII and VULGARIS $\ldots \ldots \ldots \ldots \ldots \ldots \ldots$

BIRD OF PARADISE, yellow and red

BLUE SPIREA, blue $\ldots \ldots \ldots \ldots \ldots \ldots \ldots$ BUCKEYE, white flrs

CR APE MYRTLE, pink or crimson.

DLCK PLANT, red flrs. . . . . . . . .

GLOBE FLOWER, yellow .......... GOLDEN BELL. yellow

HIBISCUS, various colors

INDIGO TREE Purple and cold....

LILAC, white, lilac and purple......

MOCK ORANGE, white

SALVIA, white and crimson $\ldots \ldots . . .$.

SMIOKE TREE. pink

SOLANUM XANTI, blue

SPIREAS, white or pink $\ldots \ldots \ldots \ldots$ i2-13

TAMIARIX, pink ...................

TURKS CAP. scarlet

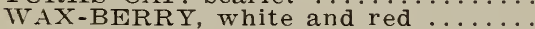

WEIGELA, pink, deep crimson or rose

\section{Evergreen Shrubs, flowering}

ACACIA ARMATA. CULTRIFORMIS, FARNESIANA, HISPIDA, and VER-

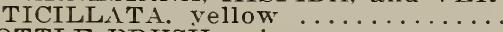

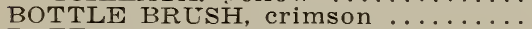
BUTTERFLY BUSH, purple .............. BROOM, vellow

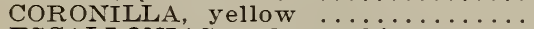

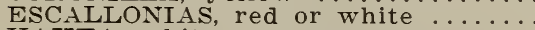
HAKEA, white

IFXICAN ORANGE, white .... MELALEUCA, white, pink, and yellow

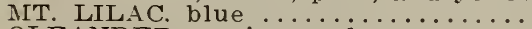
OLEANDER, various colors .......... POLYGALA, deep rose

PRIVTT, I, JAPONICA, white...

TRIV

TEA TREE, white $\ldots \ldots \ldots \ldots \ldots \ldots$
VERONICA, blue, purple and white..

\section{Evergreen Shrubs and Trees: foliage} effect.

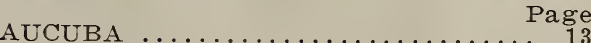

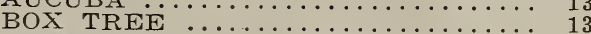

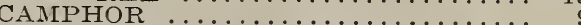

COPROSMA (coast region) .......... 14

EUONYMIUS JAPONICA, in var. ...... 14

LAUREL, in var. ................ 15

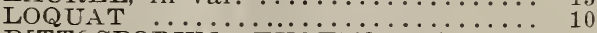

PITTUSPORUII EUGENOIDES and NIGRICANS $\ldots \ldots \ldots \ldots \ldots \ldots \ldots \ldots \ldots \ldots$
HOTINIA $\ldots \ldots \ldots \ldots \ldots \ldots \ldots$

$\underset{\text { PRIVET }}{\text { PHOTINIA } \ldots \ldots \ldots \ldots \ldots \ldots \ldots \ldots \ldots \ldots \ldots} 11$

Deciduous Shrubs and Trees-foliage effect

APPLE, NEIDWITZKIANA ....... 8

BARBERRY, purple leaved ....... 12

ELDER .................. 8

MAPLES $\ldots \ldots \ldots \ldots \ldots \ldots \ldots \ldots \ldots \ldots \ldots$

PLUMS $\ldots \ldots \ldots \ldots \ldots \ldots \ldots \ldots \ldots \ldots$

\section{Evergreen Flowering Trees}

ACACIAS, yellow ............ 9

EUCALYPTUS SIDEROXYLON-ROSEA and LEUCOXYLON-ROSEA, pink flrs. Hardy .................. 10

E. FICIFOLIA, crimson flrs., tender.. 10

E. LINEARIS, very fine graceful foliage, white flrs. ............. 10

HAKEA. White $\ldots \ldots \ldots \ldots \ldots \ldots \ldots 10$

MAJTRONE $\ldots \ldots \ldots \ldots \ldots \ldots \ldots \ldots \ldots$

SILKY OAK, orange red ......... 11

\section{Deciduous Ornamental Trees}

ACACIA NEMIU, pink flrs. ........ S

ASH, ARIZONA $\ldots \ldots \ldots \ldots \ldots \ldots$ s

CHINA TREE, yellow flrs. ....... \&

$\mathrm{LARCH} \ldots \ldots \ldots \ldots \ldots \ldots \ldots \ldots \ldots$

LINDEN $\ldots \ldots \ldots \ldots \ldots \ldots \ldots \ldots \ldots$

LOCUST, pink and crimson flrs...... 8

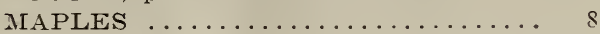

MT. ASH $\ldots \ldots \ldots \ldots \ldots \ldots \ldots \ldots \ldots$

PLANE $\ldots \ldots \ldots \ldots \ldots \ldots \ldots \ldots \ldots \ldots$

OAKS .................... -10

\section{Vines or Climbers.}

Among the quickest growing are:

AAIPELOPSIS QUINQUIFOLIA ..... 16

BIGNONIA TWEEDIANA and VIOLA-

CEA .................... 16

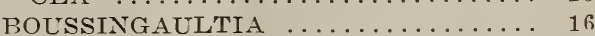

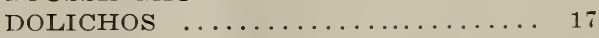

HEDERA ................ 17

JASMINE OFFICINALE ........ 17

LONICERA JAPONICA . . . . . . 17

MANDEVILLA .............. 17

MUEHLENBECKIA $\ldots \ldots \ldots \ldots \ldots \ldots \ldots .17$

PASSIFLORA ............. 17

VITIS HENRYI $\ldots \ldots \ldots \ldots \ldots \ldots \ldots \ldots, 17$

WISTERIA ................ 17

These lists are but partial, and with hardly an exception, unless so noted, include varieties that will succeed almost anywhere in California, in partial shade or in full sun. 


$$
.00
$$

